\title{
In detail explanation for the "Proof of the \\ Twin Prime Conjecture" and its applications to thermal physics
}

\author{
K.H.K. Geerasee Wijesuriya \\ Research Scientist in Physics and Astronomy \\ PhD student in Astrophysics/Cosmology
}

BSc (Hons) in Physical Science, Faculty of Science, University of

Colombo, Sri Lanka

geeraseew@gmail.com 


\section{Content}

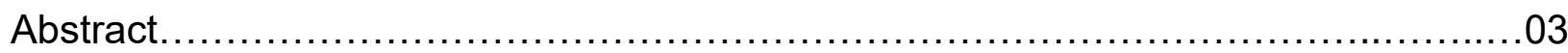

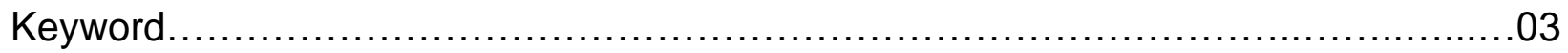

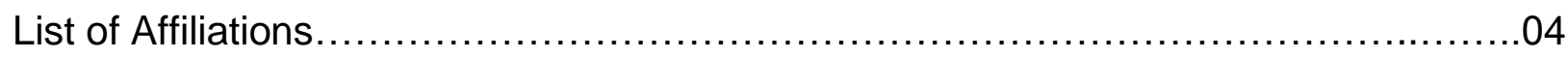

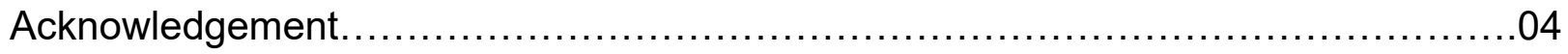

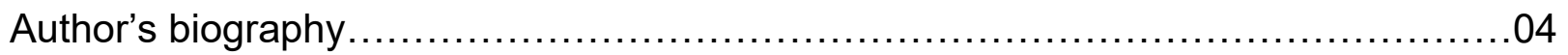

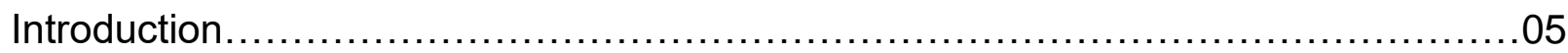

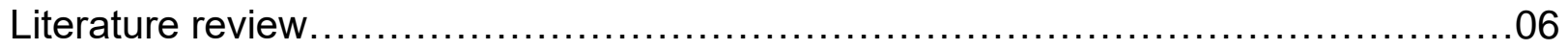

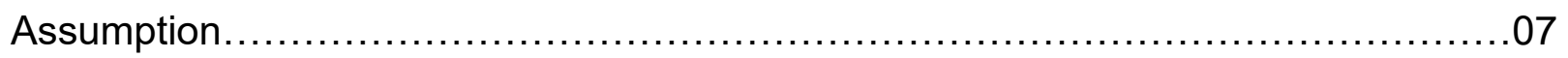

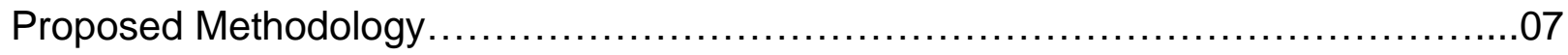

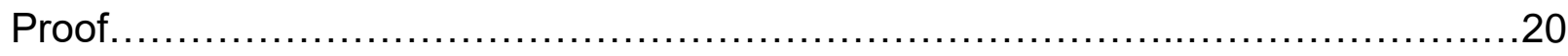

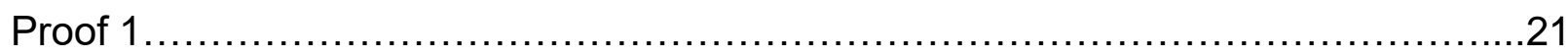

Results

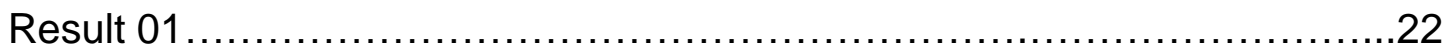

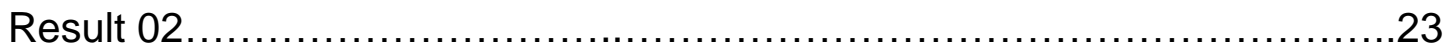

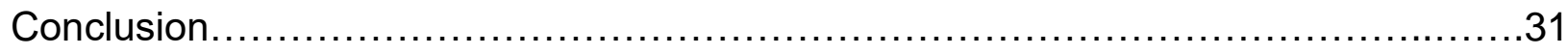

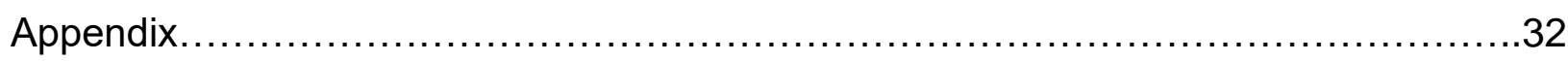

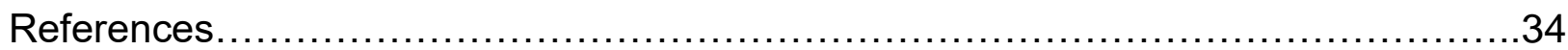




\section{Abstract}

Twin prime numbers are two prime numbers which have the difference equals to exactly 2. In other words, twin primes is a pair of two prime numbers which have the value of the difference exactly two. Sometimes the word "twin prime" is used for a pair of twin primes; an another name for this is considered as "prime twin" or called as "prime pair". Up to date there is no any exact proof/disproof for twin prime conjecture since roughly 200 years in the world. Through this research paper, my attempt is to provide a valid proof for twin prime conjecture. This new paper is the detailed explanation of my previous paper that I completed on mid of the year 2020 titled as 'Proof of Twin Prime Conjecture that can be obtained by using Contradiction method in Mathematics' (WHICH IS WELL-RECONGNIZED ALL OVER THE WORLD through researchgate

as well). And this proof of the existence of infinitely many twin primes can be applied to many subject areas in Physics, Chemistry and etc. And the proof of twin prime conjecture can be used to solve several unsolved problems in Physics, Chemistry and etc as well.

Also as an additional result, at the end of this research paper, it discusses about an application of the Proof of Twin Prime Conjecture to the Quantum and Thermal Physics. There, this research paper consider three space volumes symbolized as area A, B and C. Inside areas A and B there are microscopic particles separately. By applying the proof of the twin prime conjecture, finally this will try to conclude that although the areas $A$ and $B$ have separated by area $C$, there are some particles those have moved from the area $B$ to area $A$ (due to the high thermal pressure of area $B$ ).

\section{Keywords}

prime; contradiction; greater than ; integer ; thermal energy; difference ; eigen-function; value; penetration 


\section{List of Affiliations}

Faculty of Science, University of Colombo, Sri Lanka.

\section{Acknowledgement}

I would be thankful to my parents who gave me the strength to go forward with mathematics and Physics knowledge and achieve my scientific goals.

\section{Author's Biography}

The author of this research paper is K.H.K. Geerasee Wijesuriya . And this proof of twin prime conjecture is completely K.H.K. Geerasee Wijesuriya's proof.

Geerasee is 32 years old and Geerasee graduated with BSc (Hons) in Physical Science from the University of Colombo, Sri Lanka in 2014. And now she is following her PhD in Astrophysics/Cosmology.

Geerasee has been received invitations from several Astronomy/Physics institutions and organizations world-wide, asking to get involve with them. Also, Geerasee has already received several invitations from some private researchers around the world asking to contribute to their researches. She worked as Mathematics tutor/Instructor at Department of Mathematics, Faculty of Engineering, University of Moratuwa, Sri Lanka. Now she is a research scientist in Physics as her career. Furthermore she has achieved several other scientific achievements already. 


\section{Introduction}

A prime number is a natural number which divides only by 1 and it self. For an example, the number 2 divides by 1 and 2 only. That means 2 is divisible by 1 and itself only. But 3 is also a prime number. Because 3 divides by 1 and itself only. But 6 is not a prime number. Because 6 is divisible by 1, 2, 3 and itself. Therefore 6 is not a prime number. But any even number greater than 2, divisible by 1,2 and itself at least. Therefore any even number greater than 2 is not a prime number. Let's consider the numbers 3 and 5 . Then 3 is divisible by 1 and itself only. Also 5 is divisible by 1 and itself only. Therefore 3 and 5 both of them are prime numbers. But the difference between 5 and 3 is $5-3=2$. Therefore when we consider 5 and 3 , both of them are prime numbers but the difference between those two prime numbers is 2 . Therefore such couple of two prime numbers we called as 'Twin Prime' numbers.

With this mathematical proof, I use the contradiction method to prove that there are infinitely many twin prime numbers.

If we use the contradiction method in some mathematical result, we initially assume the negation of the requited result. Then I proceed with the mathematical steps with the usage of the well verified mathematical results. Also I should use the assumption that I assumed initially. Then after series of mathematical steps, I will ended up with a contradiction. But I know that in order to get the final contradiction, I didn't use any invalid mathematical result except our initial assumption. Therefore I can conclude that the only issue is our initial assumption. Therefore it is possible to conclude that our initial assumption is wrong. Therefore mathematically I can conclude that the negation of our initial assumption is correct. Because in mathematics, we use only the propositions. That means I can say we got the required result finally. That's how mathematical contradiction method works.

And with the 'result 1' content of this research paper, this will apply the result that 'there are infinitely many twin prime numbers', to experimental research frame with separate areas $A, B$ and $C$ to get a physical result regarding particle penetration from the area $B$ to area $A$. 


\section{Literature Review}

The question of whether there exist infinitely many twin primes is an unsolved mathematics problem in number theory since hundreds years. With this proof of twin prime conjecture, this states that there are infinitely many primes $p_{0}$ such that $p_{0}+2$ number is also prime number. In 1849, a reputed mathematician named as de Polignac made the better general conjecture that for all natural number $q$, there are infinitely many primes $p$ such that $p+2 q$ is also prime number. But when the case, which is $q=1$ of de Polignac's conjecture, is the twin prime conjecture actually.

On April 17, 2013, Yitang Zhang (a well known and reputed and famous mathematician) showed a proof that, for some integer $\mathrm{N}$ that is less than 70 million, there are infinitely many pairs of primes that differ by 70 million or less than that successfully at that time. And his paper was published with a mathematics journal named as "Annals of Mathematics" in early May 2013. Terence Tao who is also a well reputed mathematician, also proposed a Polymath Project collaborative in order to optimize Zhang's bound. On April 14, 2014, which is one year later exactly from the previously mentioned date, the bound has been decreased to 246. Moreover, by considering the Elliott-Halberstam conjecture and its generalized form, the Polymath project states that the bound has been deducted to 12 and 6 , respectively finally. These developed bounds has been found using another different path that was simpler than the previously mentioned mathematician Zhang's important discovery, and has been discovered separately by the mathematicians named as James Maynard and Terence Tao.

Although there are lots of applications to thermal physics from the subject of 'Quantum Mechanics', still thermal physics hasn't any application from the result of 'Twin Prime Conjecture' to it, since the twin prime conjecture hasn't proved before. Therefore the application of twin prime conjecture to thermal physics will be innovative. 


\section{Assumption}

Let's assume that there are finitely many twin prime numbers

Therefore we proceed by considering that there are finitely many twin prime numbers. Then let the highest twin prime numbers are $P_{R-1}$ and $\left(P_{R-1}+2\right)$. Then for all prime numbers $P_{N}$ greater than $\left(P_{R-1}+2\right),\left(P_{N}+2\right)$ is not a prime number. Where $N$ and $n$ denote two distinct natural numbers. And $\mathrm{P}_{\mathrm{N}}$ denotes the $\mathrm{N}^{\text {th }}$ prime number. $\mathrm{P}_{\mathrm{N}-1}$ denotes $(\mathrm{N}-1)^{\text {th }}$ prime number. And $\mathrm{P}_{\mathrm{N}+1}$ denotes $(\mathrm{N}+1)^{\text {th }}$ prime number. But $\mathrm{P}_{\mathrm{N}-1}>$ $\left(P_{R-1}+2\right)$. But in the below content, I use only the symbol ' $N$ ' to denote prime numbers. That means $\mathrm{P}_{\mathrm{N}}, \mathrm{P}_{\mathrm{N}-1}, \mathrm{P}_{\mathrm{N}+1}$ are considered as prime numbers. But in the below content, I don't put any particular value for ' $N$ '. That means $P_{3}$ is NOT generated as $\mathrm{N}=3, \mathrm{P}_{\mathrm{L}}$ is NOT generated as $\mathrm{N}=\mathrm{L}$. That means in the below content I use only the symbol ' $\mathrm{N}$ ' to denote the prime numbers. i.e. Only $\mathrm{P}_{\mathrm{N}}, \mathrm{P}_{\mathrm{N}+1}, \mathrm{P}_{\mathrm{N}-1}$ are considered as prime numbers and I don't put $\mathrm{N}=3$ or $\mathrm{N}=\mathrm{L}$ or $\mathrm{N}=\mathrm{Q}$ or any particular symbol as $\mathrm{N}$ to symbolize prime numbers. And in the below proof, the symbol ' $P$ ' also uses to denote a prime number. That means I use only the symbol ' $N$ ' to denote the prime numbers $P_{N}$, $\mathrm{P}_{\mathrm{N}+1}, \mathrm{P}_{\mathrm{N}-1}$ (I do not use any particular value for $\mathrm{N}$ to symbolize any prime number). But in the below proof, the symbol ' $P$ ' also uses to denote a prime number (In the below proof, $\mathrm{P}=\mathrm{P}_{\mathrm{N}-1}$ or $\mathrm{P}_{\mathrm{N}+1}$ ).

\section{Proposed Methodology}

Here in the methodology, I consider some integers with some special properties as below. And after that, I use our above assumption (1) and apply the assumption (1) to those integer numbers with the chosen special properties. Then this will try to get a contradiction. But to get the contradiction, this does not use any unverified mathematical theories. Therefore if this is capable to get a contradiction finally, it is possible to conclude that 'the only issue is our initial assumption' (1). Since our assumption (1) is a proposition, if so, it is possible to conclude that the negation of assumption (1) is true. That's how this mathematics proof will be working. 
Now let's consider some integers with the below mentioned properties and features.

Let $P_{n}$ is an odd number greater than 1 . But let $P_{3}$ is an odd natural number (greater than 1) which is divisible by $x_{3}$. But $x^{2}{ }_{3}$ does not divide $P_{3}$. Where $x_{3}$ is also an odd natural number greater than 1 . But should consider that that the chosen $x_{3}$ gives us that "[ $\left.\left(x_{3}-1\right) / 2\right]$ is an odd number".

And let we choose $P_{n}$ such that $P_{n}=\left[(M+4) / x_{3}\right]-\left(D_{0} \cdot P_{3} / x_{3}\right)$; for some odd natural number $D_{0}$, which is not equal to zero. But $\left(D_{0}+2\right)$ is divisible by $x_{3}$. But $\left(D_{0}+2\right)$ is not divisible by $x^{2}$. But we must have chosen $D_{0}$ natural number such that $x^{2}{ }_{3} \mid\left(P L . x_{3}+\left(D_{0}+2\right)\right)$ for the non-zero natural number $P_{L}$

( Where, according to the below later content, $P_{L}=(P-4) / P_{3}=$ non zero natural number )

(To know the meaning of $\mathrm{M}$, please refer the below content)

${ }^{* * *}$ And we have chosen a natural number $M$ such that $\left\{\left[(M+4) / x_{3}\right]-\left(D_{0} \cdot P_{3} / x_{3}\right)\right\}$ $\left(=P_{n}\right)$ is not divisible by $P_{3}$, but greater than 1

${ }^{* * *}$ But we must have non zero integer $t$ such that $P_{3}=(M+4) .\left[\left(x_{3}-1\right) / 2 t\right]$; where $t$ is an integer not equals to zero and also $x^{2}{ }_{3} \mid(t-1)$

To see the meaning of $t$ and $M$, please refer the below content.

\{

Let's show that the conditions in (3) and in (4) both are possible simultaneously in the proof as below:

Let we choose odd non zero integer ' $a$ ' such that ' $a$ ' is not divisible by $x_{3}$. Let choose integer $P_{Q}$ such that $P_{Q}=a+a \cdot x_{3}{ }^{2}$ (Then $P_{Q}$ is not divisible by $x_{3}$ since ' $a$ ' is not divisible by $\left.x_{3}\right)$. And ' $a$ ' is an odd number. And $x_{3}{ }^{2}$ is also an odd number. Thus $\left(a \cdot x_{3}{ }^{2}\right)$ is an odd number. But the addition of two odd numbers creates an even number. Thus $\left(a+a \cdot x_{3}{ }^{2}\right)$ is an even number. Then $P_{Q}\left(=a+a \cdot x_{3}{ }^{2}\right)$ is an even number. Then $x_{3} \cdot\left(a+a \cdot x_{3}{ }^{2}\right)=$ $(M+4)$ is an even number. i.e. $(M+4)$ is an even integer. 
But we should know that we choose odd natural number $\mathrm{D}_{0}$, which as not equals to zero. But $\left(D_{0}+2\right)$ should divisible by $x_{3}$. But $\left(D_{0}+2\right)$ is not divisible by $x^{2}{ }_{3}$. But we have chosen $D_{0}$ natural number such that $x^{2}{ }_{3} \mid\left(P_{L} . x_{3}+\left(D_{0}+2\right)\right)$ for the non-zero natural number $P_{L}$. Also we should get $P_{Q}$ such that $\left[P_{Q}-D_{0} .\left(P_{3} / x_{3}\right)\right]$ is not divisible by $P_{3}$, for the already chosen $D_{0}$. And $P_{Q}=(M+4) / x_{3}$.

Let choose an integer $t(\neq 0)$ such that $t=\left(P_{Q} / a\right)$.

But then $(\mathrm{t}-1)=\left(\mathrm{PQ}_{\mathrm{Q}} / \mathrm{a}\right)-1=\left[\left(\mathrm{PQ}_{\mathrm{Q}}-\mathrm{a}\right) / \mathrm{a}\right]=\mathrm{x}_{3}{ }^{2}\left(\right.$ since $\left.\mathrm{t}=\mathrm{PQ}_{\mathrm{Q}} / \mathrm{a}\right)$. That means $(\mathrm{t}-1)$ $=\mathrm{x}_{3}{ }^{2}$. Therefore $(\mathrm{t}-1)$ is divisible by $\mathrm{x}_{3}{ }^{2}$.

But $(M+4)=P_{Q} \cdot X_{3}$. Then $[(M+4) / 2 . t]=P_{Q} \cdot X_{3} / 2 t=P_{Q} \cdot X_{3} \cdot a /\left(2 \cdot P_{Q}\right)=a \cdot X_{3} / 2$ (6)

(because $\left.\mathrm{t}=\mathrm{PQ}_{\mathrm{Q}} / \mathrm{a}\right)$.

And we can consider the integer $P_{3}(\neq 0)$ as $P_{3}=(M+4) \cdot\left[\left(x_{3}-1\right) / 2 \cdot t\right]=\left(x_{3}-1\right) \cdot a \cdot x_{3} / 2$ (= integer, since $\left(x_{3}-1\right)$ is an even number) (by using the result (6))

( Because $\left(x_{3}-1\right) \cdot a \cdot x_{3} / 2$ is divisible by $x_{3}$. But since ' $a$ ' is not divisible by $x_{3}$, $\left[\left(x_{3}-1\right) \cdot a / 2\right]$ is not divisible by $x_{3}$. That means $P_{3}$ is not divisible by $x_{3}{ }^{2}$. And ' $a$ ' is an odd number. Also $\left[\left(x_{3}-1\right) / 2\right]$ is an odd number according to our initial choice of $x_{3}$. And $x_{3}$ is also an odd integer. Thus [( $\left.\left.x_{3}-1\right) \cdot a_{1} x_{3} / 2\right]$ is an odd integer. Then by considering all of those things, the term $(M+4) \cdot\left[\left(x_{3}-1\right) / 2 \cdot t\right]=\left[\left(x_{3}-1\right) \cdot a \cdot x_{3} / 2\right]$ satisfies the required conditions to be the value of $P_{3}$. Thus we can say that we can write $\left.P_{3}=(M+4) \cdot\left[\left(x_{3}-1\right) / 2 \cdot t\right]=\left(x_{3}-1\right) \cdot a \cdot x_{3} / 2\right)$.

But please remember that $2 \mid\left(x_{3}-1\right)$ and $\left[\left(x_{3}-1\right) / 2\right]$ is an odd integer. ${ }^{* *}$ See the Proof 1 below to see the existence of $\left(P_{N}-2\right)=P_{3} \cdot x_{3}$ such that $P_{3}=$ a. $x_{3}$. $\left[\left(x_{3}-1\right) / 2\right]$ for some odd integer ' $a$ ' ; where $\left[\left(x_{3}-1\right) / 2\right]$ is an odd integer.

\{

But we should get for the chosen $P_{Q},\left[P_{Q}-D_{0} .\left(P_{3} / x_{3}\right)\right]$ is not divisible by $P_{3}$. 
Let's prove that as below: $P_{n}=P_{Q}-D_{0} \cdot\left(P_{3} / x_{3}\right)=a+a \cdot x_{3}^{2}-D_{0} \cdot\left(x_{3}-1\right) \cdot a / 2$ $=\left(a \cdot x_{3} / 2\right) \cdot\left[2 \cdot x_{3}+\left(D_{0}+2\right) / x_{3}-D_{0}\right]$. Let $\left(D_{0}+2\right) / x_{3}=d ; d$ is a natural number which is not divisible by $x_{3}$.

Then $P_{n}=\left(a . x_{3} / 2\right) .\left[2 . x_{3}+d-D_{0}\right] \ldots \ldots . .(7.1)$

\{

Let $\left[2 . x_{3}+d-D_{0}\right]=\left(x_{3}-1\right) \cdot K^{\prime}$. Where assume $K^{\prime}$ as an odd integer number.

Then $\left(\mathrm{D}_{0}-\mathrm{K}^{\prime}\right)$ is an even number. But $5 .\left(\mathrm{D}_{0}+2\right)$ is an odd number.

Then 5. $\left(D_{0}+2\right) \neq a^{\prime} .\left(D_{0}-K^{\prime}\right)^{2}$ for any integer $a^{\prime}$, (including $a^{\prime}=\left(a^{2} 0-1\right)$ for $a_{0}$ an integer ).

Then

$\mathrm{D}_{0}(+/-)\left[\left(\mathrm{D}_{0}-\mathrm{K}^{\prime}\right)^{2}+5 \cdot\left(\mathrm{D}_{0}+2\right)\right]^{(1 / 2)}=\mathrm{D}_{0}(+/-)\left(\mathrm{D}_{0}-\mathrm{K}^{\prime}\right) \cdot \mathrm{f}^{(1 / 2)}$

$f^{(1 / 2)}$ is not an integer. Here, $f$ is an integer. But $f^{(1 / 2)}$ is an irrational number.

Let $g^{\prime}=\left(D_{0}-K^{\prime}\right) \cdot f^{(1 / 2)}=g^{\prime}=$ an irrational number.

Then $\left[\left(D_{0}-K^{\prime}\right)^{2}+4 \cdot\left(D_{0}+2\right)\right]=\left(D_{0}-K^{\prime}\right)^{2} \cdot f-\left(D_{0}+2\right)$

$=\left[\left(D_{0}-K^{\prime}\right) \cdot f^{(1 / 2)}-\left(D_{0}+2\right)^{(1 / 2)}\right] \cdot\left[\left(D_{0}-K^{\prime}\right) \cdot f^{(1 / 2)}+\left(D_{0}+2\right)^{(1 / 2)}\right]$

Here $f^{(1 / 2)}$ is an irrational number.

$\left[\left(D_{0}-K^{\prime}\right) \cdot f^{(1 / 2)}-\left(D_{0}+2\right)^{(1 / 2)}\right] \cdot\left[\left(D_{0}-K^{\prime}\right) \cdot f^{(1 / 2)}+\left(D_{0}+2\right)^{(1 / 2)}\right]$

$=\left(D_{0}-K^{\prime}\right)^{2} \cdot f-\left(D_{0}+2\right)$. Let $\left(D_{0}-K^{\prime}\right)^{2} \cdot f-\left(D_{0}+2\right)=E^{2} \ldots \ldots(7) "$

Then $f-\left(D_{0}+2\right) /\left(D_{0}-K^{\prime}\right)^{2}=\left[E /\left(D_{0}-K^{\prime}\right)\right]^{2}$

Since $K^{\prime}$ and $D_{0}$ are odd integers, $\left(D_{0}-K^{\prime}\right)^{2}$, is an even number.

Thus, $\left(D_{0}+2\right) /\left(D_{0}-K^{\prime}\right)^{2}$ is not an integer. Since $f$ is an integer, by (7)'":

$\left[E /\left(D_{0}-K^{\prime}\right)\right]^{2}$ is not an integer. Thus $\left[E /\left(D_{0}-K^{\prime}\right)\right]$ is not an integer.......((7) $)^{\prime \prime \prime \prime}$

By $(7)^{\prime}$, since $\mathrm{K}^{\prime}$ is an arbitrary odd integer, $\mathrm{f}$ is an arbitrary integer. Thus by (7)": $\mathrm{E}$ is an arbitrary integer. Thus by (7)'"': for all such arbitrary $E,\left[E /\left(D_{0}-K^{\prime}\right)\right.$ ] is not an integer 
means, $E$ is not an integer. Thus by (7)": [ ( $\left.\left.D_{0}-K^{\prime}\right)^{2} \cdot f-\left(D_{0}+2\right)\right]$ is not a squared number.

Thus $\left[\left(D_{0}-K^{\prime}\right) \cdot f^{(1 / 2)}-\left(D_{0}+2\right)^{(1 / 2)}\right] \cdot\left[\left(D_{0}-K^{\prime}\right) \cdot f^{(1 / 2)}+\left(D_{0}+2\right)^{(1 / 2)}\right]$ is not a squared number. But $\left[\left(D_{0}-K^{\prime}\right) \cdot f^{(1 / 2)}-\left(D_{0}+2\right)^{(1 / 2)}\right] \cdot\left[\left(D_{0}-K^{\prime}\right) \cdot f^{(1 / 2)}+\left(D_{0}+2\right)^{(1 / 2)}\right]=$ $\left[\left(D_{0}-K^{\prime}\right)^{2}+4 .\left(D_{0}+2\right)\right]$. Thus $\left[\left(D_{0}-K^{\prime}\right)^{2}+4 \cdot\left(D_{0}+2\right)\right]$ is not a squared number. Thus $\left[\left(D_{0}-K^{\prime}\right)^{2}+4 .\left(D_{0}+2\right)\right]^{(1 / 2)}$ is an irrational number. Let $z=D_{0}(+/-)\left[\left(D_{0}-K^{\prime}\right)^{2}+4 .\left(D_{0}+2\right)\right]^{(1 / 2)}$; where $z$ is an irrational number. Then $z=D_{0}(+/-)\left[\left(D^{2} 0+K^{\prime 2}-2 \cdot D_{0} \cdot K^{\prime}+4 \cdot D_{0}+8\right]^{(1 / 2)}\right.$ $=\left\{2 \cdot D_{0}(+/-)\left[\left(4 \cdot D^{2} 0+4 \cdot\left[K^{\prime 2}-2 \cdot D_{0} \cdot K^{\prime}+4 \cdot D_{0}+8\right]\right]^{(1 / 2)}\right\} / 2\right.$ Then $z^{2}-2 \cdot D_{0} \cdot z-\left[K^{\prime 2}-2 \cdot D_{0} \cdot K^{\prime}+4 \cdot D_{0}+8\right]=0$ Then $z^{2}=K^{\prime 2}-2 \cdot D_{0} \cdot K^{\prime}+2 \cdot D_{0} \cdot(z+2)+8$

Then $z^{2}-2 \cdot D_{0} \cdot z=4+4 \cdot D_{0}+4+K^{\prime} .\left(K^{\prime}-2 \cdot D_{0}\right)$

Then $D^{2}{ }_{0}+z^{2}-2 \cdot D_{0} \cdot z=D^{2} 0+4+4 \cdot D_{0}+4+K^{\prime} .\left(K^{\prime}-2 \cdot D_{0}\right)$

Then $\left(D_{0}-z\right)^{2}=\left(D_{0}+2\right)^{2}+4+K^{\prime} .\left(K^{\prime}-2 \cdot D_{0}\right) \ldots \ldots(7.1 .1)$

Since $z$ is not an integer number, $\left(D_{0}-z\right)^{2}$ is not a square number.

Therefore, $\left[\left(D_{0}+2\right)^{2}+4+K^{\prime} .\left(K^{\prime}-2 \cdot D_{0}\right)\right]^{(1 / 2)}$ is not an integer number.

By (7.1.1): $\left\{\left(K^{\prime}-D_{0}\right)(+/-)\left[\left(D_{0}+2\right)^{2}+4+K^{\prime} .\left(K^{\prime}-2 . D_{0}\right)\right]^{(1 / 2)}\right\} /\left[2 .\left(K^{\prime}-2\right)\right]$ is not an integer.

If $x_{3}=\left\{\left(K^{\prime}-D_{0}\right)(+/-)\left[\left(D_{0}+2\right)^{2}+4+K^{\prime} .\left(K^{\prime}-2 \cdot D_{0}\right)\right]^{(1 / 2)}\right\} /\left[2 .\left(K^{\prime}-2\right)\right]:$

$x^{2} 3-\left[\left(K^{\prime}-D_{0}\right) /\left(K^{\prime}-2\right)\right] . x_{3}-\left[\left(D_{0}+2\right) /\left(K^{\prime}-2\right)\right]=0$. Then $x_{3}$ is not an integer number.

Then $x^{2}{ }_{3} \cdot\left(K^{\prime}-2\right)=K^{\prime} \cdot x_{3}+\left(D_{0}+2\right)-x_{3} \cdot D_{0}$

Then, $x_{3} .\left(K^{\prime}-2\right)=K^{\prime}+\left[\left(D_{0}+2\right) / x_{3}\right]-D_{0}$

Then 2. $x_{3}+d-D_{0}=K^{\prime} .\left(x_{3}-1\right) ; d=\left(D_{0}+2\right) / x_{3} \ldots \ldots . .(7.1 .2)$ 
Then if $K^{\prime}$ is an (odd) integer, $x_{3}$ is not an integer. But $x_{3}$ is an integer. Therefore our assumption, which is: $K^{\prime}$ is an (odd) integer is not valid. Therefore it is possible to conclude that $\mathrm{K}^{\prime}$ is not an (odd) integer.

(But since $P_{n}=\left(a \cdot x_{3} / 2\right)$. [2. $x_{3}+d-D_{0}$ ] and since $P_{3}=a \cdot x_{3} \cdot\left(x_{3}-1\right) / 2$ and since $P_{n}$ is an odd integer, $\mathrm{K}^{\prime}$ can't be an even integer).

Therefore $\mathrm{K}^{\prime}$ is not an even/odd integer. Therefore $\mathrm{K}^{\prime}$ is not an integer.

Therefore it is possible to conclude that by (7.1) and (7.1.2):

$P_{n}$ is not divisible by $P_{3} \ldots \ldots \ldots . .(7.2)$

$$
\text { \} }
$$

Then we have the non-zero integer number $P_{Q}\left(=a+a \cdot x^{2}{ }_{3}\right)$ such that $\left[P_{Q}-D_{0} \cdot\left(P_{3} / x_{3}\right)\right]$ $\left(=P_{n}\right)$ is not divisible by $P_{3}$. By (8): the given $P_{n}$ in (8) is still not divisible by $P_{3} \ldots \ldots . . .(08)$

But we know that by $(7)$, we have $P_{3}(\neq 0)$ such that $P_{3}=(M+4) \cdot\left[\left(x_{3}-1\right) / 2 . t\right]$.

That means $t=(M+4) .\left[\left(x_{3}-1\right) / 2 \cdot P_{3}\right]$.

That means by (5), (7) and (8):

when we choose the integer $t$ such that $t=(M+4) \cdot\left[\left(x_{3}-1\right) /\left(2 \cdot P_{3}\right)\right.$ whenever $(t-1)=x^{2}{ }_{3}$ , it is possible to have $P_{Q}\left(=a+a \cdot x^{2}{ }_{3}\right)$ such that $\left[P_{Q}-D_{0} \cdot\left(P_{3} / x_{3}\right)\right]$ is not divisible by $P_{3}$

That means, when there is the integer $t$ such that $t=(M+4) \cdot\left[\left(x_{3}-1\right) /\left(2 \cdot P_{3}\right)\right.$ whenever $(t-1)=x^{2}{ }_{3} \rightarrow$ we have $P_{Q}\left(=a+a \cdot x^{2}{ }_{3}\right)$ such that $\left[P_{Q}-D_{0} \cdot\left(P_{3} / x_{3}\right)\right]$ is not divisible by $\mathrm{P}_{3}$

Thus by considering the statements in (3) and (4), it is possible to say: the conditions in (3) and in (4) both are possible simultaneously in the proof

$$
\text { \} }
$$

But $P_{N}$ is an arbitrary prime number greater than $\left(P_{R-1}+2\right)$. Because there are infinitely many prime numbers. And here $\left(P_{N}-2\right)>\left(P_{R-1}+2\right)$. Thus $\left(P_{N}-2\right)$ is not a prime number. 
Where each arbitrary $\mathrm{P}_{\mathrm{N}}$ prime number obey (that means we choose a set of $\mathrm{P}_{\mathrm{N}}$ ( > $\left(P_{R-1}+2\right)$ arbitrarily such that each arbitrarily chosen $P_{N}$ give $\left.u s\right)$ :

" $P_{3}$ is divisible by $x_{3}$. But $x^{2}{ }_{3}$ does not divide $P_{3}$; whenever $\left(P_{N}-2\right)=P_{3} . x_{3}$ "

And here $\left(P_{N}+2\right)>\left(P_{R-1}+2\right)$. Then according to our assumption, $\left(P_{N}+2\right)$ is also not a prime number.

Since $P_{N}$ is a prime number, we already know that $\left(P_{N}-2\right)$ is also not a prime number (Since $P_{N}-2>P_{R-1}+2$ )

Then for the integer $P_{3}$ greater than 1 such that $\left(P_{N}-2\right) / P_{3}=x_{3}$; where $x_{3}$ is an integer greater than 1. But here we have already considered that $x_{3} \mid P_{3}$. But $x_{3}^{2}$ does not divide $P_{3}$. Here either $P_{3}$ or $x_{3}$ not equals to $\left(P_{N}-2\right)$.

Then $\left(P_{N}-2\right)=P_{3}{ }^{*} x_{3}$

But $\left(P_{N}+2\right), P_{n}$ both are odd numbers. Thus $\left(P_{N}+2\right)=P_{n}+2 . I$; for some $/$ integer (where $I \neq 0)$

Then $\left(P_{N}-2\right)=P_{n}+2 . I-4=P_{n}+2 .(I-2)$

And we know that $\left(P_{N}+2\right)=P_{n}+2 . l \rightarrow P_{N}=P_{n}+2 . l-2$

Thus by (15): $P_{n}+2 . I-2=P_{N}$. Thus by (12) and (15): $P_{3}{ }^{*} x_{3}+2=P_{n}+2 . I-2$

Thus $\mathrm{P}_{3}{ }^{*} \mathrm{x}_{3}-2 . I+4=\mathrm{Pn}_{\mathrm{n}}$

Thus $P_{3}{ }^{*} X_{3}+2 .(I-2)=P_{n}+4 .(I-2)=P_{n}+2 \cdot P_{N}-4-2 \cdot P_{n}=2 \cdot P_{N}-4-P_{n}($ by $(14)$ )

Thus $P_{3}{ }^{*} x_{3}+2 .(I-2)=2 \cdot P_{N}-4-P_{n}=P_{n}$.

Thus $P_{3}{ }^{*} x_{3}+2 .(I-2)=P_{n}{ }^{\prime}=2 \cdot P_{3}{ }^{*} x_{3}-P_{n}$

Thus $P_{3}{ }^{*} x_{3}+2 . I=4+2 . P_{3}{ }^{*} x_{3}-P_{n}$

Then $P_{3}{ }^{*} x_{3}+(2 . I+M)=\left(4+M-P_{n}\right)+2 . P_{3}{ }^{*} x_{3} ;$ Where $M$ is an integer $(M \neq 0)$

Then $(2 . I+M)=\left(4+M-P_{n}\right)+P_{3}{ }^{*} x_{3} ;$ Where $M$ is an integer $\neq 0$.

But we chose $M$ such that $(M+4)$ is divisible by $x_{3}$. But $(M+4)$ is not divisible by $x^{2}{ }_{3}$ 
But by (7): $P_{3}=(M+4) \cdot\left[\left(x_{3}-1\right) / 2 . t\right]$. If $x_{3}=3$, then $(M+4) / P_{3}=t$. If $x_{3}=3, P_{3}$ divides $(M+4)$

But we know that $P_{3}$ is divisible by $x_{3}$. But $x^{2}{ }_{3}$ does not divide $P_{3}$. And we know that $\left(P_{3}{ }^{*} x_{3}\right)$ is divisible by $x_{3}$. And we know that $P_{n}$ is not divisible by $P_{3}$

Thus by (18), (19) and (20):

$\mathrm{P}_{3}$ does not divide $(2 . I+\mathrm{M})$

But $P_{N}$ is an arbitrary prime greater than $\left(P_{R-1}+2\right)$. Then let $\left\{\left(P_{N}+A_{1}\right), P_{N}\right\}$ are two arbitrary consecutive primes set such that each primes are greater than $\left(P_{R-1}+2\right)$. Where each arbitrary $P_{N}$ prime number obey:

" $P_{3}$ is divisible by $x_{3}$. But $x^{2}{ }_{3}$ does not divide $P_{3}$; whenever $\left(P_{N}-2\right)=P_{3} . x_{3}$ "

Here since $P_{N}>\left(P_{R-1}+2\right)$ and since $\left(P_{N}+A_{1}\right)>\left(P_{R-1}+2\right), A_{1} \neq(+/-) 2$. Because we have assumed that there are finitely many twin prime numbers. Then there is a highest twin primes (which are considered as $\mathrm{P}_{\mathrm{R}-1}$ and $\mathrm{P}_{\mathrm{R}-1}+2$ ). Then for any arbitrarily chosen consecutive two prime numbers greater than $\left(\mathrm{P}_{\mathrm{R}-1}+2\right)$, the difference between them is greater than 2.

But now choose two particular two consecutive primes (greater than $\left(P_{R-1}+2\right)$ ) from the arbitrary prime number set $\left\{\left(P_{N}+A_{1}\right), P_{N}\right\}$ such that those chosen two particular consecutive primes obey: $\left[P_{3} \mid\left(A_{1}-2\right)\right]$ and $P_{N}>A_{1}$. i.e. where particularly we choose $A_{1}$ such that $P L-X_{3}=B_{2}$. Where $\left(A_{1}-2\right) / P_{3}=B_{2}$. Where $P L=(P-4) / P_{3}$ (later I have proven that $P_{L}$ is an integer). Here $P=$ chosen particular prime $\left(P_{N}+A_{1}\right)$. Since $A_{1} \neq-2$ for all primes $\left\{P_{N},\left(P_{N}+A_{1}\right)\right\}$ (Greater than $\left.P_{R-1}+2\right)$, we can have such particularly chosen two primes $\left\{P_{N},\left(P_{N}+A_{1}\right)\right\}$, which are obeying $\left[P_{3} \mid\left(A_{1}-2\right)\right]$ (because $P_{3}$ does not divide -4 . Because if $A_{1}=-2$, then the expression $\left[P_{3} \mid\left(A_{1}-2\right)\right] \equiv$ $\left[P_{3} \mid-4\right]$. But it is not valid. But since $A_{1} \neq-2$ for all primes $\left\{P_{N},\left(P_{N}+A_{1}\right)\right\}$, there is no any issue with that expression $\left[P_{3} \mid\left(A_{1}-2\right)\right]$. And also according to recent discoveries done by number theory mathematicians, they have proven that there are infinitely many consecutive prime number couples, with the difference between each two prime numbers (in each couple), which is less than the value of 
247. But since we know about twin primes greater than 247, when assuming the assumption (1) above, we know $P_{N}>247$. But as mentioned above, there are infinitely many consecutive prime number couples with $A_{1}<247$. Therefore it is possible to conclude that there exists such a particular prime number couple in the prime number set $\left\{\left(P_{N}+A_{1}\right), P_{N}\right\}$ such that $P_{N}>A_{1}$. And refer the 'Proof' below to see the existence of two consecutive primes $\left(P_{N}+A_{1}\right)$ and $P_{N}$ such that $\left.P_{3} \mid\left(A_{1}-2\right)\right]$. And refer 'Proof 1' to see the existence of an integer $\left(P_{N}-2\right)$ such that $\left(P_{N}-2\right)=P_{3 .} x_{3}$ such that $P_{3}$ is divisible by $x_{3}$. But $x^{2}{ }_{3}$ does not divide $P_{3}$.

And $P_{N}$ and $\left(P_{N}+A_{1}\right)$ are two consecutive two primes numbers greater than $\left(P_{R-1}+2\right)$. Thus here $A_{1} \neq(+/-) 2$, since there are finite number of twin primes according to our assumption. Because the difference between two consecutive two primes (greater than $\left.P_{R-1}+2\right)$ is greater than 2. BUT REMEMBER THAT $P_{N}$ AND $\left(P_{N}+A_{1}\right)$ ARE CONSECUTIVE PRIMES greater than $\left(P_{R-1}+2\right)$ which obey $P_{3}$ is divisible by $x_{3}$. But $x^{2}{ }_{3}$ does not divide $P_{3}$; whenever $\left(P_{N}-2\right)=P_{3} . x_{3}$.

\{

Here $\left(P_{N}-2\right)=P_{3} X_{3}$ and $\left(P_{N}+A_{1}\right)=P=$ Prime. That means $P_{3} X_{3}+\left(A_{1}+2\right)=$ $P \ldots \ldots(a)$

And $\left(A_{1}-2\right)$ is divisible by $P_{3}$. But $P_{3}$ does not divide 4 . Thus $\left(A_{1}+2\right)$ is not divisible by $\mathrm{P}_{3}$.

But since $P_{3}{ }^{*} x_{3}$ is divisible by $P_{3}$, by (a): $P$ is not divisible by $P_{3}$

But $\left(A_{1}-2\right)$ is divisible by $P_{3}$ and since $\left(x_{3} \mid P_{3}\right), x_{3} \mid\left(A_{1}-2\right)$. But $x_{3}$ does not divide 4 since $x_{3}$ is an odd number (since $\left.\left(P_{N}-2\right)=P_{3} \cdot x_{3}\right)$. Thus $\left(A_{1}+2\right)$ is not divisible by $x_{3}$. But since $P_{3}{ }^{*} x_{3}$ is divisible by $x_{3}$, by (a): $P$ is not divisible by $x_{3}$

Therefore according to above steps as in (b) and (c), we can write $P_{3} X_{3}+\left(A_{1}+2\right)=P$ as a prime

\}

But $(2 . I+M)=P_{N}-P_{n}+2+M=\left(P_{N}+A_{1}\right)+\left(M+2-A_{1}-P_{n}\right)$ 
By (19): $x_{3} \mid(M+4)$. But $\left[P_{3} \mid\left(A_{1}-2\right)\right]$.

But since $\left[P_{3} \mid\left(P_{N}-2\right)\right]$ and since $P_{3}$ does not divide $\left(A_{1}+2\right),\left\{\left(A_{1}+2\right)+\left(P_{N}-2\right)\right\}$ does not divide by $P_{3}$. i.e. $P\left(=\left(P_{N}+A_{1}\right)\right)$ does not divide by $P_{3}$. Thus our choice of $A_{1}$ such that $\left[P_{3} \mid\left(A_{1}-2\right)\right]$ is okay (because the expression $P_{3} \mid\left(P_{N}-2\right)$ implies that $P_{3}$ does not divide $P$ ). Then still I can state that $P$ is as a prime number.

But $\left[P_{3} \mid\left(P_{N}-2\right)\right]$ and $\left[P_{3} \mid\left(A_{1}-2\right)\right]$. Thus $\left(P_{N}-2\right)=P_{3} . X_{3}$ and $\left(A_{1}-2\right)=P_{3} . B_{2} ;$ where $X_{3}$ and $B_{2}$ are integers and each of them not equals to 0 .

Thus $\left(P_{N}+A_{1}-4\right)=\left(P_{N}-2\right)+\left(A_{1}-2\right)=P_{3} \cdot x_{3}+P_{3} \cdot B_{2}=(P-4)$

i.e $P_{3} \mid(P-4) \ldots \ldots \ldots \ldots \ldots(25)$ Therefore, $P L=(P-4) / P_{3}=$ natural number.

Let's consider $M$ integer such that $M=P-C$; for some integer ' $C$ ' $\neq 0$ (26)

Because we can have relationship between any two integers by using another introducing integer.

But by (19): $x_{3} \mid(M+4)$ and $P_{3} \mid(P-4)$ by $(25)$.

By (26): $P=(M+C)$. Thus by (25): $[(M+C)]-4=P_{3} . P L \ldots \ldots \ldots . .(27)$

Where $P L=\left[(P-4) / P_{3}\right]=$ integer, but not equals to 0 . Because $P$ is a prime number (which not equals to zero).

Then $\left(P_{L} / x_{3}\right)=\left[(P-4) /\left(x_{3} P_{3}\right)\right]=(P-4) /\left(P_{N}-2\right)$

$=\left[\left(P_{N}-2\right)+\left(A_{1}-2\right)\right] /\left(P_{N}-2\right)=1+\left[\left(A_{1}-2\right) /\left(P_{N}-2\right)\right]\left(\right.$ because $\left.P=P_{N}+A_{1}\right)$

If $A_{1}<0$, then either $1+\left[\left(A_{1}-2\right) /\left(P_{N}-2\right)\right]<0$ or $0<1+\left[\left(A_{1}-2\right) /\left(P_{N}-2\right)\right]<1$.

But $P_{L}=\left[(P-4) / P_{3}\right]$. Since $(P-4)>0$ and since $x_{3}>1, P_{3}>1 ; P_{L}>0$. Thus it is impossible to have $1+\left[\left(A_{1}-2\right) /\left(P_{N}-2\right)\right]<0$. Thus if $A_{1}<0$, we have $0<1+\left[\left(A_{1}-2\right) /\left(P_{N}-2\right)\right]<1$. Then $\left(P_{L} / X_{3}\right)$ is not an integer, since $\left(P_{L} / X_{3}\right)=1+\left[\left(A_{1}-2\right) /\left(P_{N}-2\right)\right]$. 
If $A_{1}>0$, then $A_{1}>2$. Because the difference between the considered two consecutive primes $\left(=A_{1}\right)$ is greater than 2. Then $0<\left(P_{N}-A_{1}\right)<\left(P_{N}-2\right)$ (because for the chosen particular primes $\left.\left\{P_{N},\left(P_{N}+A_{1}\right)\right\} ; P_{N}>A_{1}\right)$.

Then $\left(P_{L} / x_{3}\right)=1+\left[\left(A_{1}-2\right) /\left(P_{N}-2\right)\right]=1+\left[\left(A_{1}-P_{N}+P_{N}-2\right) /\left(P_{N}-2\right)\right]$

$=2+\left[\left(A_{1}-P_{N}\right) /\left(P_{N}-2\right)\right]=2-\left[\left(P_{N}-A_{1}\right) /\left(P_{N}-2\right)\right]$.

Since $A_{1} \neq(+/-) 2$ and by $(28):\left[\left(P_{N}-A_{1}\right) /\left(P_{N}-2\right)\right]$ is not an integer.

Then $2-\left[\left(P_{N}-A_{1}\right) /\left(P_{N}-2\right)\right]=\left(P_{L} / X_{3}\right)$ is not an integer.

Then for $A_{1}>0$ and for $A_{1}<0:\left(P_{L} / X_{3}\right)$ is not an integer.

Thus here $\mathrm{x}_{3}$ does not divide $\mathrm{P}$

Then $\left(P_{L} / x_{3}\right)=1+\left[\left(A_{1}-2\right) /\left(P_{N}-2\right)\right]=1+\left[\left(A_{1}-P_{N}+P_{N}-2\right) /\left(P_{N}-2\right)\right]$

$=2+\left[\left(A_{1}-P_{N}\right) /\left(P_{N}-2\right)\right]=2-\left[\left(P_{N}-A_{1}\right) /\left(P_{N}-2\right)\right]$.

Since $A_{1} \neq(+/-) 2$ and by $(28):\left[\left(P_{N}-A_{1}\right) /\left(P_{N}-2\right)\right]$ is not an integer.

Then $2-\left[\left(P_{N}-A_{1}\right) /\left(P_{N}-2\right)\right]=\left(P_{L} / X_{3}\right)$ is not an integer.

Then for $A_{1}>0$ and for $A_{1}<0:\left(P_{L} / x_{3}\right)$ is not an integer.

Thus here $\mathrm{x}_{3}$ does not divide $\mathrm{P}$

But $\left[(M+4) / x_{3}\right]=P_{Q}=$ integer, but not equals to 0 .

Thus by (27): $\left[\left(x_{3} \cdot P_{Q}-4+C\right)\right]-4=P_{3} . P L$

Thus $C-4=\left[\left(P_{3} \cdot P L+4\right)-x_{3} \cdot P_{Q}\right]$. Thus $C=\left[\left(P_{3} \cdot P_{L}+8\right)-x_{3} \cdot P_{Q}\right]$ (30)

By (23): $(2 . I+M)=\left(P_{N}+A_{1}\right)+\left(M+2-A_{1}-P_{n}\right)=P+\left(M+2-A_{1}-P_{n}\right)$

$=P+P-C+2-A_{1}-P_{n}=2 \cdot P-C-P_{3} \cdot B_{2}-P_{n}$ ..(31) (because $\mathrm{M}=\mathrm{P}-\mathrm{C}$

and $\left.A_{1}-2=P_{3} . B_{2}\right)$

By (30): $C=\left[\left(P_{3} \cdot P_{L}+8\right)-x_{3} \cdot P_{Q}\right]$.

Then 2.P $-C-P_{n}=2 \cdot P+x_{3} \cdot P_{Q}-\left(P_{3} \cdot P L+8\right)-P_{n}$ 
$=2 \cdot(P-4)+x_{3} \cdot P_{Q}-P_{n}-\left(P_{3} \cdot P_{L}\right)=2 \cdot P_{3} \cdot P_{L}+x_{3} \cdot P_{Q}-P_{n}-\left(P_{3} \cdot P_{L}\right)$

$=P_{3} \cdot P_{L}+\left[x_{3} \cdot P_{Q}-P_{n}\right]$ (because $P-4=P_{3} \cdot P_{L} ;$ for non-zero integer $\left.P_{L}\right)$

Then 2.P $-C-P_{n}=x_{3} . P_{3} \cdot\left\{\left(P_{L} / x_{3}\right)+\left[\left(P_{Q} / P_{3}\right)-\left(P_{n} /\left(x_{3} . P_{3}\right)\right)\right]\right\}$

But we chose $P_{n}$ such that $\left(x_{3} . P_{n}\right)=(M+4)-D_{0} \cdot P_{3} ;$ for some integer $D_{0}$.

${ }^{* * *}$ But we have chosen $D_{0}$ integer such that $x^{2}{ }_{3} \mid\left(P L . X_{3}+\left(D_{0}+2\right)\right)$. Where $D_{0} \neq 0$ and $\left(D_{0}+2\right)$ is divisible by $x_{3}$ (it implies that $D_{0}$ is not divisible by $\left.x_{3}\right)$. But $\left(D_{0}+2\right)$ is not divisible by $\mathrm{x}^{2}{ }_{3}$.

And therefore here $\left(P_{L} / x_{3}\right)+\left[\left(D_{0}+2\right) / x^{2}{ }_{3}\right]=D^{\prime}$ for some integer $D^{\prime}$.

Since $P L$ is not divisible by $x_{3}$, there exists such an integer number $\left(D_{0}+2\right)$ (not equals to zero), such that $x^{2}{ }_{3} \mid\left(P L . x_{3}+\left(D_{0}+2\right)\right)$; whenever $\left(2+D_{0}\right)$ is not divisible by $x^{2}{ }_{3}$.

Then $P_{n}=\left[(M+4) / x_{3}\right]-\left(D_{0} \cdot P_{3} / x_{3}\right)$. Then $\left[\left(P_{Q} / P_{3}\right)-\left(P_{n} / P_{3}\right)\right]=\left(D_{0} / x_{3}\right)$

$=\left[x_{3} \cdot\left(P_{Q} / P_{3}\right)-\left(P_{n} / P_{3}\right)\right]-\left(x_{3}-1\right) \cdot\left(P_{Q} / P_{3}\right)$.

Then $\left[\mathrm{x}_{3} .\left(\mathrm{PQ}_{\mathrm{Q}} / \mathrm{P}_{3}\right)-\left(\mathrm{P}_{\mathrm{n}} / \mathrm{P}_{3}\right)\right]=\left(\mathrm{D}_{0} / \mathrm{x}_{3}\right)+\left(\mathrm{x}_{3}-1\right) .\left(\mathrm{PQ}_{\mathrm{Q}} / \mathrm{P}_{3}\right)$

Then $\left[\left(P_{Q} / P_{3}\right)-\left(P_{n} /\left(x_{3} . P_{3}\right)\right)\right]=\left(D_{0} / x^{2}{ }_{3}\right)+\left(x_{3}-1\right) \cdot\left(P_{Q} /\left(x_{3} . P_{3}\right)\right)=$

$\left(D_{0} / x^{2}{ }_{3}\right)+\left(x_{3}-1\right) \cdot\left(P_{Q} /\left(x^{2} 3 \cdot u\right)\right)=\left[D_{0} \cdot u+\left(x_{3}-1\right) \cdot P_{Q}\right] /\left(u \cdot x^{2}{ }^{2}\right)$

where $u \cdot x_{3}=P_{3}$, and $u$ is a non-zero integer and then $u$ is not divisible by $x_{3}$, since $x_{3}{ }^{2}$ does not divide $\mathrm{P}_{3}$.

But by (4): we chose $\left(t . P_{3}\right)=(M+4) .\left[\left(x_{3}-1\right) / 2\right] ; t$ is an integer $\neq 0$ such that $x^{2}{ }_{3} \mid(t-1)$.

Then $\left.\left(P_{3} / x_{3}\right)=u=(M+4) .\left(x_{3}-1\right)\right] /\left(2 . t . x_{3}\right)=$ integer

Then $\left.u=P_{Q} \cdot\left(x_{3}-1\right)\right] / 2 . t$

Thus by (33): $\left[\left(P_{Q} / P_{3}\right)-\left(P_{n} /\left(x_{3} . P_{3}\right)\right)\right]=\left[D_{0} \cdot u+2 . t . u\right] /\left(u . x^{2}{ }_{3}\right)=\left(D_{0}+2 t\right) / x^{2}{ }_{3}$; where $P_{Q}$ is not divisible by $x_{3}$. Then according to the formula for $u$, still ' $u$ ' is not divisible by $\mathrm{X} 3$. 
Then $\left\{\left(P_{L} / x_{3}\right)+\left[\left(P_{Q} / P_{3}\right)-\left(P_{n} /\left(x_{3} . P_{3}\right)\right)\right]\right\}=\left(P_{L} / x_{3}\right)+\left[\left(D_{0}+2 t\right) / x^{2}{ }_{3}\right]$

$=\left(P L / x_{3}\right)+\left[\left(D_{0}+2\right) / x^{2}{ }_{3}\right]+\left[2 .(t-1) / x^{2}{ }_{3}\right]=\left[\left[P L . x_{3}+\left(D_{0}+2\right)\right] / x^{2}{ }_{3}\right]+t^{\prime}=D^{\prime}=$ integer number ( Because by $(7)$ : $t^{\prime}=2 .(t-1) / x^{2}{ }_{3}=$ integer).

Since $P_{L}$ is not divisible by $x_{3}$, there exists such an integer number $D_{0}$ (not equals to zero), such that $x^{2}{ }_{3} \mid\left(P_{L} . x_{3}+\left(D_{0}+2\right)\right)$. Whenever $\left(2+D_{0}\right)$ is divisible by $x_{3}$. Whenever $\left(2+D_{0}\right)$ is not divisible by $x^{2} 3$.

Then by (32): 2.P $-C-P_{n}=P_{3} . x_{3}\left[D^{\prime}\right]$; where $\left(P_{L} / x_{3}\right)$ and $\left(D_{0}+2\right) / x^{2}{ }_{3}$ are not integers (by 29). But we chose $D_{0}$ such that $x^{2}{ }_{3} \mid\left(P_{L} \cdot x_{3}+\left(D_{0}+2\right)\right)$. Then 2.P $-C-P_{n}=$ $P_{3} . x_{3}$. D' ;

where $D^{\prime}=\left(P L . x_{3}+\left(D_{0}+2\right)\right) / x^{2}{ }_{3}+t^{\prime}=$ integer, but not equals to 0 .

Then $P_{3} \mid\left(2 . P-C-P_{n}\right)$

by (31), (34): $P_{3} \mid(2 . I+M)$

(35) (since in (31), $B_{2}$ is a non-zero integer)

In (35), there is no any particular value for $x_{3}$. But it is possible to state that $x_{3}=3$ in (35) with the same result: $P_{3} \mid(2 . I+M)$ (since $3\left(=x_{3}\right)$ obeys the required conditions to be $x_{3}=3$, since $(3-1) / 2$ is an odd number.

But by (22) (when $x_{3}$ is 3 ): $P_{3}$ does not divide $(2 . I+M)$

Thus by (35) and (36): We have a contradiction

But in order to get the contradiction as in (37), I used only the well-established mathematical results and equations and our initial assumption (1). Therefore the only possibility that may cause the final contradiction (37) is: our assumption (1) is false. Since the assumption (1) is a proposition, according to the definition of a 'proposition' in mathematics, we can say that the negation of the initial assumption (1) is true. But the negation of our assumption (1) is: There are infinitely many twin prime numbers. Therefore as the result of above mathematical contradiction method, it is possible to state that "there are infinitely many Twin Prime Numbers". 


\section{Proof}

Let's prove that there exists consecutive primes $P_{N}$ and $\left(P_{N}+A_{1}\right)$ such that [ $P_{3} \mid\left(A_{1}-2\right)$ ] for some odd integer $P_{3}$ which is greater than 1 (when there exist consecutive prime numbers $P_{N}$ and $\left(P_{N}+A_{1}\right)$ which both are greater than $\left.\left[P_{R-1}+2\right]\right)$ through this 'Proof' as below.

By $2^{\text {nd }}$ reference: $\mathrm{P}_{\mathrm{N}-1}=\left(\mathrm{P}_{\mathrm{N}}+\mathrm{A}_{1}\right)=2+\sum_{j=1}^{N-2} h j$, where $h_{\mathrm{j}}=\mathrm{P}_{\mathrm{j}+1}-\mathrm{P}_{\mathrm{j}}$ for all $\mathrm{j} \in\{1,2, .$. $(\mathrm{N}-2)\}$ or $\mathrm{P}_{\mathrm{N}+1}=\left(\mathrm{P}_{\mathrm{N}}+\mathrm{A}_{1}\right)=2+\sum_{j=1}^{N} h j$ when $\mathrm{j} \epsilon\{1,2, \ldots, \mathrm{N}\}$. Here $\left(\mathrm{P}_{\mathrm{N}}+\mathrm{A}_{1}\right)=\mathrm{P}_{\mathrm{N}+1}$ or $P_{N-1}$, depends on the sign of $A_{1}$.

$\mathrm{P}_{\mathrm{N}-1}=\mathrm{P}_{\mathrm{N}}+\mathrm{A}_{1}=2+\sum_{j=1}^{N-2} h j\left(\right.$ when $\left.\mathrm{A}_{1}<0\right)$. If $\mathrm{A}_{1}>0, \mathrm{P}_{\mathrm{N}+1}=\mathrm{P}_{\mathrm{N}}+\mathrm{A}_{1}=2+\sum_{j=1}^{N} h j$

Consider the case that $\mathrm{A}_{1}<0$.

Then $\left(\mathrm{A}_{1}-2\right)=-\mathrm{P}_{\mathrm{N}}+\sum_{j=1}^{N-2} h j$.

Then $\left(2-\mathrm{A}_{1}\right)=\mathrm{P}_{\mathrm{N}}-\sum_{j=1}^{N-2} h j$

But by $2^{\text {nd }}$ reference: for all $€>0$, there is a natural number ' $m$ ' such that for all $(N-2)>$ $\mathrm{m} ; \mathrm{h}_{\mathrm{N}-2}<\mathrm{P}_{\mathrm{N}-2} . €$

Let $\epsilon_{s}$ is a positive real number $\epsilon_{s}=\left[-B+C_{s}+k^{\prime}+P_{N}+B_{2} . P_{3}\right] / P_{s}>0$, such that $h_{s}$ $<P_{s}{ }^{*} \epsilon_{s}$ for all $s>(N-3)$. Let here the chosen $\epsilon_{s}$ implies that $m=(N-3)$ (Here $s$ is going from 1 to $(N-2)$. Then " for all $s>(N-3)$ " means $s \equiv(N-2)$. Where $k$ ' is an integer number which not equals to 0 and we choose $k^{\prime}$ such that $k^{\prime} /(N-2)$ is an integer. Here the chosen $k^{\prime}$ integer number is responsible for $h_{s}<P_{s}{ }^{*} \epsilon_{s}$ for all $s>$ $(\mathrm{N}-3)$ (i.e. $s=\mathrm{N}-2$ ) and $\mathrm{k}^{\prime}$ is responsible for $\epsilon_{s}>0$ as well. That means here the value of $k^{\prime}$ is responsible to say " $\epsilon_{s}$ is existing such that $h_{s}<P_{s}{ }^{*} \epsilon_{s}$, for $s=(N-2)$ ".

Here $h_{j}=b_{j}-\left[k^{\prime} /(N-2)\right]$ for all $j<(N-2)=s$. And where $\Sigma b_{j}=B$ for $j<(N-2)=s$. Then for some $C_{s}, h_{s}=P_{s}{ }^{*} \epsilon_{s}-C_{s}$; here $s \equiv(N-2) .{ }^{* * *}$ the meaning of ' $j$ ' is the order number and $h_{j}$ is the (prime) gap between $P_{j+1}$ and $P_{j}$. Please refer the below content and the $2^{\text {nd }}$ reference. But here we chose $\mathrm{C}_{\mathrm{N}-2}$ such that $\mathrm{h}_{\mathrm{N}-2}=\mathrm{P}_{\mathrm{N}-2}{ }^{*} \epsilon_{\mathrm{N}-2}-\mathrm{C}_{\mathrm{N}-2}$ 
But $h_{N-2}=P_{N-2}{ }^{*} \epsilon_{N-2}-C_{N-2}=\left(-B+k^{\prime}+P_{N}+B_{2}\right.$. $\left.P_{3}\right)$. Where $k^{\prime}$ is a non zero integer number. Now let's use the $2^{\text {nd }}$ reference to proceed further. By (38):

$\left(\mathrm{A}_{1}-2\right)=-\mathrm{P}_{\mathrm{N}}+\sum_{j=1}^{N-2} h j=-\mathrm{P}_{\mathrm{N}}+\left(-\mathrm{B}+\mathrm{k}^{\prime}+\mathrm{P}_{\mathrm{N}}+\mathrm{B}_{2} \cdot \mathrm{P}_{3}\right)+\mathrm{B}-(\mathrm{N}-2) \cdot\left[\mathrm{k}^{\prime} /(\mathrm{N}-2)\right]$

$=\mathrm{B}_{2} \cdot \mathrm{P}_{3}$

Thus by (39): $\left(A_{1}-2\right)=P_{3} . B_{2}$. Thus there exist consecutive prime numbers $P_{N}$ and $\left(P_{N}+A_{1}\right)$ both greater than $\left(P_{R-1}+2\right)$ such that $\left(A_{1}-2\right)=B_{2} \cdot P_{3} ;$ for some integer $B_{2}$ $(\neq 0)$.

Similar to above, if $A_{1}>0$, we can proceed with the similar steps to prove that $\left(A_{1}-2\right)=$ $B_{2 .} P_{3} ;$ for some integer $B_{2}(\neq 0)$ when $A_{1}>0$.

\section{Proof 1}

Let's prove the existence of an integer $\left(P_{N}-2\right)\left(>P_{R-1}+2\right)$ such that $\left(P_{N}-2\right)=P_{3} . x_{3}$ such that $P_{3}$ is divisible by $x_{3}$. But $x^{2}{ }_{3}$ does not divide $P_{3}$ as below.

Where $P_{3}=(a / 2) \cdot x_{3} .\left(x_{3}-1\right)$ for some odd integer 'a'. And where $\left[\left(x_{3}-1\right) / 2\right]$ is an odd natural number. Here $x_{3}=3$.

By $2^{\text {nd }}$ reference: $\mathrm{P}_{\mathrm{N}}=2+\sum_{j=1}^{N-1} g j \quad$, where $\mathrm{g}_{\mathrm{j}}=\mathrm{P}_{\mathrm{j}+1}-\mathrm{P}_{\mathrm{j}}$ for all $\mathrm{j} \epsilon\{1,2, \ldots \ldots, \mathrm{N}-1\}$

Then $\left(\mathrm{P}_{\mathrm{N}}-2\right)=\sum_{j=1}^{N-1} g j$

But by $2^{\text {nd }}$ reference: for all $€>0$, there is a natural number 'mo' such that for all $\mathrm{N}>$ $m_{0} ; g_{N}<P_{N} . \epsilon$.

Let $\epsilon_{s}$ is a positive real number $\epsilon_{s}=\left[-A+C_{s}+x^{2}{ }_{3} \cdot k_{1}\right] / P_{s}>0$, such that $h_{s}<P_{s}{ }^{*} \epsilon_{s}$ for all $s>(N-2)$. Let here the chosen $\epsilon_{s}$ implies that $m_{0}=(N-2)$ (Here $s$ is going from 1 to $\mathrm{N}-1$. Then " for all $s>(\mathrm{N}-2)$ " means $s \equiv(\mathrm{N}-1))$. Where $\mathrm{k}_{1}$ is an integer number which is not divisible by $x_{3}$. And let $k_{1}=a .\left(x_{3}-1\right) / 2$. Where ' $a$ ' is an odd integer which is not divisible by $x_{3}$. And $\left[\left(x_{3}-1\right) / 2\right]$ is an odd natural number. Here the chosen $P_{Q}$ and $t$ integer numbers $(\neq 0)$ are responsible for $g_{s}<P_{s}{ }^{*} \epsilon_{s}$ for all $s>(N-2)$ (i.e. $s=N-1$ ) and $\epsilon_{s}>0$. OR in another words, the previously chosen integer $M$ and $t$ are responsible 
to say that above fact. That means here the value of $k_{1}$ (or in another words, ' $a$ ') is responsible to say " $\epsilon_{s}$ is existing such that $g_{s}<P_{s}{ }^{*} \epsilon_{s}$, for $s=N-1 "$. Here $g_{j}=a_{j}$ for all $\mathrm{j}<(\mathrm{N}-1)=\mathrm{s}$. And where $\Sigma \mathrm{aj}_{\mathrm{j}}=\mathrm{A}$ for $\mathrm{j}<(\mathrm{N}-1)=\mathrm{s}$. Then for some $\mathrm{C}_{\mathrm{s}}$, $g_{s}=P_{s}{ }^{*} \epsilon_{s}-C_{s}$; here $s \equiv(N-1) .{ }^{* * *}$ the meaning of ' $j$ ' is the order number and $g_{j}$ is the prime gap between $P_{j+1}$ and $P_{j}$. Please refer the below content and the $2^{\text {nd }}$ reference. But here we chose $\mathrm{C}_{\mathrm{N}-1}$ such that $\mathrm{g}_{\mathrm{N}-1}=\mathrm{P}_{\mathrm{N}-1}{ }^{*} \epsilon_{\mathrm{N}-1}-\mathrm{C}_{\mathrm{N}-1}$. And where $\left[\left(x_{3}-1\right) / 2\right]$ is an odd natural number.

But $g_{N-1}=\left(-A+x^{2} 3 \cdot k_{1}\right)$. Now let's use the $2^{\text {nd }}$ reference to proceed further.

By (40): $\left(\mathrm{P}_{\mathrm{N}}-2\right)=\sum_{j=1}^{N-1} g j$. But $\sum_{j=1}^{N-1} g j=\mathrm{A}+\left(-\mathrm{A}+\mathrm{x}^{2}{ }_{3} \cdot \mathrm{k}_{1}\right)=\mathrm{x}^{2}{ }_{3} \cdot \mathrm{k}_{1}$

Thus by (40) and (41): $\left(P_{N}-2\right)=x^{2}{ }_{3} . k_{1}$; where $k_{1}$ is not divisible by $x_{3}$. Then $\left(P_{N}-2\right)=$ $\mathrm{x}_{3} .\left(\mathrm{x}_{3} . \mathrm{k}_{1}\right)=\mathrm{x}_{3} . \mathrm{P}_{3}$; where $\mathrm{P}_{3}$ is divisible by $\mathrm{x}_{3}$. But $\mathrm{P}_{3}$ is not divisible by $\mathrm{x}^{2}{ }_{3}$. And where $\left[\left(x_{3}-1\right) / 2\right]$ is an odd natural number. Thus $\left(P_{N}-2\right)=P_{3}$. $x_{3}$; where $P_{3}$ is divisible by $x_{3}$. But $P_{3}$ is not divisible by $x^{2}{ }_{3}$. Where $\left[\left(x_{3}-1\right) / 2\right]$ is 1 . Thus there exists an integer set $\left\{\left(P_{N}-2\right)=P_{3}\right.$. $x_{3}$ : where $P_{3}$ is divisible by $x_{3}$ but $P_{3}$ is not divisible by $x^{2}{ }_{3}$ and where [ $\left(x_{3}-1\right) / 2$ ] is an odd natural number equals to 1 \} such that $P_{N}$ is an arbitrary prime number greater than $\left(\mathrm{P}_{\mathrm{R}-1}+2\right)$. Whenever $\mathrm{x}_{3}=3,\left(\mathrm{x}_{3}-1\right) / 2$ is an odd number. Thus applying $x_{3}=3$ is okay (since for the above proof $x_{3}$ is arbitrary, whenever $\left(x_{3}-1\right) / 2$ is an odd number).

\section{Results}

\section{Result 01}

Therefore I have used our assumption (1) to get the contradiction finally, as showed in (37). Therefore it is possible to conclude that our assumption (1) is false. But we know that our assumption (1) is a proposition. Thus it is possible to say that the negation of the assumption (1) is true. But the negation of our assumption (1) is: There are infinitely many twin prime numbers. Thus there are infinitely many twin prime numbers. 


\section{Result 02}

With this result 02, I am intending to apply our above main result to the thermal physics researches. BUT PLEASE NOTE THAT THERE ARE NO ANY RELATIONSHIPS BETWEEN EACH OF THE EQUIVALENT SYMBOLS OF ABOVE METHODOLOGY AND THIS RESULT 02. That means there is no any relationship between the symbol ' $N$ ' in above methodology and in this result 02 and so on for other symbols.

By the above main result, it is possible to say that there are infinitely many twin prime numbers

Let's consider two regions symbolized as $A$ and $B$. And there are microscopic matter particles (with zero electric charge) inside the area $A$ and $B$, which move inside the areas $A$ and $B$ with the individual energies (thermal). But there is a border region (C) between the areas of $A$ and $B$. But let's consider that the region $A$ can have comparably infinitely many quantum states separately. And at the border line of the system of areas $A, B$ and $C$, the potential energy tends to infinity. Therefore particles inside $A / B / C$ can't go outside of the system $A / B / C$. Also particles those are outside of $A / B / C$ can't come inside of $A / B / C$. And the region $A$ and $B$ are comparably large separate areas. Let's figure out the situation as below.

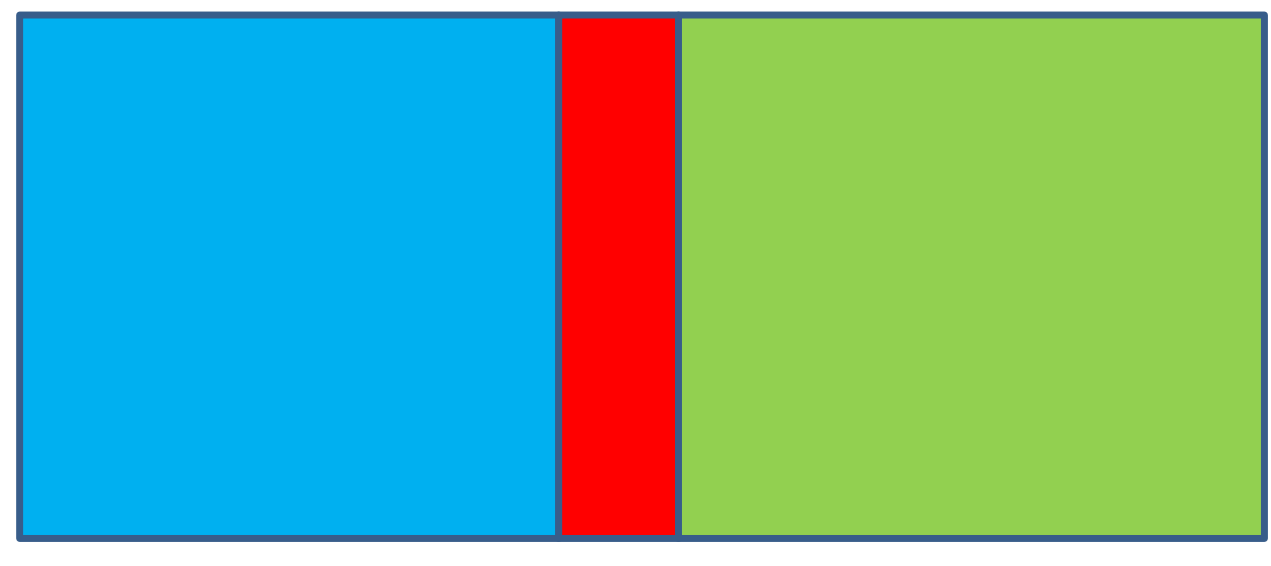

$\begin{array}{lll}\text { Region A } & \text { Region C }\end{array}$

Figure 01 (How the matter particles distributed) 
Blue color area is the region A, green color area is the region $B$ and the red color area is the region $\mathrm{C}$. But the region $\mathrm{A}$ can have comparably infinitely many quantum states separately. And area $C$ is an area which the matter particles can't cross that particular area $C$ easily and go to the opposite area. Let at time $t=0$, there are no any considerable thermal pressure of the area $\mathrm{A}$ and area $\mathrm{B}$. Also at $\mathrm{t}=0$, we allow to tend the temperature of the area $A$ and $B$ to Kelvin 0 . But during the time $t \in\left(0, t_{1}\right]$, we gradually increase the temperature of the region $B$. That means we increase the thermal pressure of the area $B$ gradually during time $t \in\left(0, t_{1}\right]$. But we do not considerably change the temperature of the region $A$ during time $t \in\left(0, t_{1}\right]$. But during the time which is before $t=0$ of the system up to $t=0$, there are no any considerable energy or the thermal pressure inside the area $A$ or $B$ that allows to cross the area $A$ or $B$ by the opposite side microscopic matter particles (which were inside A or B).

Let's consider the 3 dimensional Schrodinger equation for an arbitrarily chosen particle $(P)$ from the region $A$ (during $\left.t \epsilon\left(0, t_{1}\right]\right)$ as below:

$-\left(\hbar^{2} / 2 \mathrm{M}\right) \nabla^{2} \psi_{i}+V_{\psi_{i}}=E_{i} \psi_{i}$

Where $\mathrm{M}$ is the mass of the microscopic particle $\mathrm{P} . \mathrm{V}$ is the total potential energy of $\mathrm{P}$. $E_{i}$ is the energy eigenvalue of the ' $i$ 'th eigen state of $P$ at some time $t$ (where $t \epsilon\left(0, t_{1}\right]$ ). $\hbar$ is the Plank constant. And $\psi_{i}$ is the wave function of $P$ when it is in the ' $i$ 'th eigen state. But $P$ is an arbitrarily chosen microscopic particle.

Where $\nabla^{2}=\frac{1}{r^{2}}\left[\frac{\partial}{\partial r}\left(r^{2} \frac{\partial}{\partial r}\right)+\left(\frac{1}{\sin \theta}\right) \frac{\partial}{\partial \theta}\left(\sin \theta \frac{\partial}{\partial \theta}\right)+\frac{1}{\sin ^{2} \theta} \frac{\partial^{2}}{\partial \varphi^{2}}\right]$

Where $r$ is the distance coordinate to $P$ from the origin of the research frame. $\theta$ is the polar coordinate to $P$ from the origin of the research frame. $\varphi$ is the azimuthal coordinate to $\mathrm{P}$ from the origin of the research frame.

But it is possible to find the energy orthonormal eigen function $\left(\Psi_{i}\right)$ of ' $i$ 'th quantum state of $P$, by considering the thermal energy of $P$, other kinetic and potential energies of $P$.

But as we consider, there are infinitely many quantum states in $A$. Thus $i$ can go

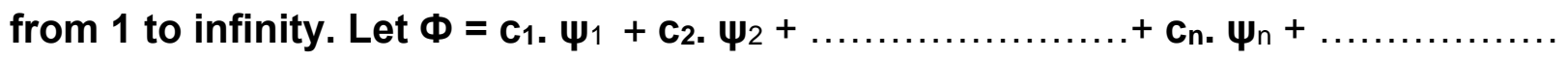


Where we consider $\boldsymbol{\Psi}_{\mathrm{i}}$ 's are as orthonormal eigen functions. i $\in\{1,2,3, \ldots \ldots \ldots, \mathrm{n}$ ...

Where $\mathrm{c}_{\mathrm{i}}$ are linearly independent constants $; \mathrm{i} \in\{1,2,3, \ldots \ldots \ldots \ldots, \mathrm{n}, \ldots \ldots \ldots \ldots\}$

Then Probability $\left(Q_{i}\right)$ of finding the particle $P$ in the quantum state ' $i$ ' $=\left|c_{i}^{2}\right|$

Let's consider that $\left|\mathbf{C i}^{2}\right|=\left\{\left(1 / \mathbf{P i}_{\mathrm{i}+1}\right)\left\{1 /\left[1-\left(\mathbf{P i}_{\mathrm{i}} / \mathbf{P}_{\mathrm{i}+1}\right)\right]\right\}\right\}^{\sqrt{\mathrm{i}}}$

Where $P_{i}$ and $P_{i+1}$ are two arbitrary natural numbers (greater than some arbitrary natural number $\mathbf{N}$ and for the considered arbitrary natural number $N$, the minimum $P_{i}$ and $P_{i+1}$ should obey $P_{i+1}-P_{i}=2$. i.e. for the chosen $N,\left[P_{2}-P_{1}\right]=2$. Since there are infinitely many twin prime numbers according to my above proof (i.e. infinitely many such $P_{1}$ and $P_{2}$ such that $\left.\left[P_{2}-P_{1}\right]=2\right)$, there exists infinitely many such natural numbers $N$ ) and for all such $P_{i+1}$ and $P_{i} ;\left[P_{i} / P_{i+1}\right]$ is a rational number for all natural numbers $P_{i+1}$ and $P_{i}$.

Since $P_{i+1}>P_{i},\left(P_{i} / P_{i+1}\right)<1$.

But $\left[\mathrm{Pi}_{\mathrm{i}} / \mathrm{P}_{\mathrm{i}+1}\right]$ is a rational number for all natural numbers $\mathrm{P}_{\mathrm{i}+1}$ and $\mathrm{P}_{\mathrm{i}}$. That means for any natural numbers $\mathrm{P}_{\mathrm{i}+1}$ and $\mathrm{P}_{\mathrm{i}}, \mathrm{P}_{\mathrm{i}+1}$ and $\mathrm{P}_{\mathrm{i}}$ haven't any common factors. Then it is possible to understand and possible to consider that $\mathrm{P}_{\mathrm{i}+1}$ and $\mathrm{P}_{\mathrm{i}}$ as two consecutive prime numbers.

But then $\left|\mathrm{Ci}^{2}\right|=1 /\left[\mathrm{P}_{\mathrm{i}+1}-\mathrm{P}_{\mathrm{i}}\right]^{\sqrt{\mathrm{i}}}$; for all 'i' th eigen state of $\mathrm{P}$.

Then the Probability $\left(Q_{i}\right)$ of finding the particle $P$ in the quantum state ' $i$ ' $=\left|c_{i}{ }^{2}\right|$ $=1 /\left[P_{i+1}-P_{i}\right]^{v_{i}}$ Then $Q_{i}=1 /\left[P_{i+1}-P_{i}\right]^{\sqrt{i}}$ for primes $P_{i+1}$ and $P_{i}$. We know that the number of quantum states inside area $A$ tends to infinity.

But $Q_{i}=1 /\left[P_{i+1}-P_{i}\right]^{\sqrt{i}}$; where $P_{i+1}$ is the ' $i+1$ ' th prime number (among the group of primes greater than some arbitrary natural number $\mathrm{N}), \mathrm{P}_{i}$ is the 'i'th prime number (among the group of primes greater than the arbitrary natural number $\mathrm{N}$ ). But $\mathrm{N}$ is greater than 2 .

But we know that there are infinitely many consecutive prime numbers couples $P_{i+1}$ and $P_{i}$ (greater than $N$ some natural number) with $P_{i+1}>\left(P_{i}+2\right)$. And by my 
above proof twin prime conjecture, it is possible to state that there are infinitely many prime numbers $P_{i+1}$ and $P_{i}$ with $P_{i+1}=\left(P_{i}+2\right)$ (each of them are greater than some natural number $\mathbf{N}$ )

Let's show that there are infinitely many consecutive prime numbers couples $\mathbf{P}_{\mathrm{i}+1}$ and $P_{i}$ (each of them are greater than some arbitrary natural number $N$ ) with $P_{i+1}>$ $\left(P_{i}+2\right)$ as below, inside this below curly brackets.

\{

We know that there are infinitely many twin prime numbers (by my initial proof in this research paper).

Let's denote those infinite number of twin primes (each of the twin primes are greater than 3 and also greater than the arbitrary natural number $N$ ) as $\left\{b_{1}, b_{2}\right\},\left\{b_{3}, b_{4}\right\}$, $\left\{b_{5}, b_{6}\right\},\left\{b_{7}, b_{8}\right\}, \ldots \ldots,\left\{b_{n-1}, b_{n}\right\},\left\{b_{n+1}, b_{n+2}\right\},\left\{b_{n+3}, b_{n+4}\right\}, \ldots \ldots \ldots \ldots$

Where above $\left\{b_{i-1}, b_{i}\right\}$ indicates twin primes couple. Symbol $i$ is a natural number.

But let's show that among above indicated $\left\{b_{i-1}, b_{i}\right\}$, taken from primes set $\left\{b_{1}, b_{2}, b_{3}\right.$, $\left.b_{4}, \ldots \ldots \ldots \ldots . . ., b_{n-2}, b_{n-1}, b_{n}, b_{n+1}, b_{n+2}, b_{n+3}, b_{n+4}, \ldots \ldots \ldots\right\}$, there are two primes $\left\{b_{m}, b_{m+1}\right\}$ such that $\left(b_{m+1}-b_{m}\right)>2$.

We know that $\left\{b_{n-1}, b_{n}\right\},\left\{b_{n+1}, b_{n+2}\right\},\left\{b_{n+3}, b_{n+4}\right\}$ are 3 consecutive twin primes set those have taken arbitrarily from the infinite number of twin primes (But here it is possible to have $b_{j}=b_{j+1}$ for some $j$ th prime $)$.

i.e. $\left[b_{n+4}-b_{n+3}\right]=2,\left[b_{n+2}-b_{n+1}\right]=2,\left[b_{n}-b_{n-1}\right]=2$

(But here it is possible to have $b_{j}=b_{j+1}$ for some $j$ th prime ).

If $b_{j} \neq b_{j+1}$ for all $b_{j}$ and $b_{j+1}$ in (42.1.1), then for some $b_{j}$ and $b_{j+1},\left[b_{j+1}-b_{j}\right] \geq 4$ (since $b_{j}$ and $b_{j+1}$ are odd numbers).

Here $j \in\{(n+4),(n+3),(n+2),(n+1), n,(n-1),(n-2)\}$.

But if all $b_{n+3}=b_{n+2}, b_{n+1}=b_{n}, b_{n-1}=b_{n-2}$ in (42.1.1), then either $b_{n+4}$ or $b_{n+3}$ or $b_{n+2}$ or $b_{n+1}$ or $b_{n}$ or $b_{n-1}$ is not a prime number. Because if $b_{k}=b_{k-1}$ for some $k$ natural number such that $k \in\{n+3, n+2, n+1, n,(n-1),(n-2)\}$ in (42.1.1), then at least one $b_{k}$ is 
divisible by 3 (because each $b_{j}$ here is greater than 3 ). Because among every 3 consecutive odd numbers, there is one odd number which is divisible by 3 (because all considering prime numbers here are odd numbers). Therefore not all $b_{n+3}=b_{n+2}, b_{n+1}=$ $b_{n}, b_{n-1}=b_{n-2}$ in (42.1.1).

Then for some prime numbers among $b_{n+4}, b_{n+3}, b_{n+2}, b_{n+1}, b_{n}, b_{n-1}$ :

$\left[b_{j+1}-b_{j}\right] \geq 4$ (since $b_{j}$ and $b_{j+1}$ are odd numbers).

By (42.1.2) and (42.1.3): for some $b_{j}$ and $b_{j+1},\left[b_{j+1}-b_{j}\right] \geq 4$. But $b_{j}$ and $b_{j+1}$ are arbitrary prime numbers.

Therefore for some primes $b_{j+1}$ and $b_{j}$ (arbitrary) : [ $\left.b_{j+1}-b_{j}\right] \geq 4$.

Since $b_{j+1}$ and $b_{j}$ are some arbitrary primes, we can say that there exists infinitely many primes $\mathrm{P}_{\mathrm{i}+1}$ and $\mathrm{P}_{\mathrm{i}}$ such that $\left[\mathrm{P}_{\mathrm{i}+1}-\mathrm{P}_{\mathrm{i}}\right] \geq 4$.

Thus there are infinitely many consecutive prime number couples $\mathbf{P}_{i+1}$ and $\mathbf{P}_{\mathrm{i}}$ with $P_{i+1}>\left(P_{i}+2\right)$. Thus there are infinitely many consecutive prime numbers couples $P_{i+1}$ and $P_{i}$ (which are greater than the arbitrary natural number $N$ ) such that $P_{i+1}>$ $\left(P_{i}+2\right)$.

\}

Now let's return to the proof after above (42.1).

Since i $\epsilon\{1,2,3, \ldots \ldots . ., n, \ldots \ldots$.$\} , we can put the symbol 'i' as below also:$

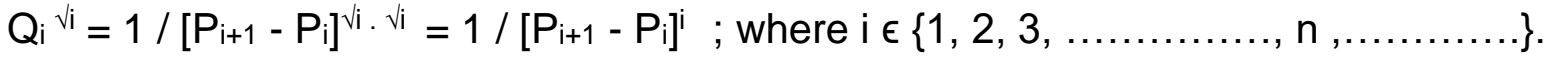

Then let's separate $1 /\left(\mathrm{P}_{\mathrm{i}+1}-\mathrm{P}_{\mathrm{i}}\right)^{\mathrm{i}}$ into two parts as below.

( Here by (42.1), it is possible to write $Q_{i}^{ل i}=1 /\left(P_{i+1}-P_{i}\right)^{i}$ for all $i \in\{1,2,3, \ldots . ., n$, ......... )

Then $Q_{i}^{\sqrt{ } i}=\left[1 /\left(q_{i+1}-q_{i}\right)\right]^{i}$ OR $Q_{i}^{\sqrt{ } i}=(1 / 2)^{i}$ $(42.1 .4)$

$\left\{\right.$ Where $\left(q_{i+1}-q_{i}\right)>2$ in the term $\sum_{i=1}^{\infty} \frac{1}{(q i+1-q i)^{i}}$ and $q_{i+1}$ and $q_{i}$ are two consecutive primes with $\left.\left(q_{i+1}-q_{i}\right)>2\right\}$ 
Then by (42.1.4): $\sum_{i=1}^{\infty}(Q i)^{\sqrt{ } i}=\sum_{i=1}^{\infty}\left(\frac{1}{(q i+1-q i)^{i}} * \delta_{\mathrm{i}}\right)+\sum_{i=1}^{\infty}\left[\left(\frac{1}{2^{i}}\right) * \delta^{\prime} i\right]$

Where $\delta_{i}=1, \delta_{i}{ }^{\prime}=0 ;\left(P_{i+1}-P_{i}\right)>2$

Where $\delta_{i}=0, \delta_{i}{ }^{\prime}=1 ;\left(P_{i+1}-P_{i}\right)=2$

Because we have infinitely many primes $q_{i+1}$ and $q_{i}$ such that $\left(q_{i+1}-q_{i}\right)>2$. Also we have infinitely many primes $\mathrm{P}_{\mathrm{i}+1}$ and $\mathrm{P}_{\mathrm{i}}$ such that $\left(\mathrm{P}_{\mathrm{i}+1}-\mathrm{P}_{\mathrm{i}}\right)=2$ (by 42.1 )

Where $\left(q_{i+1}-q_{i}\right)>2$ in the term $\sum_{i=1}^{\infty} \frac{1}{(q i+1-q i)^{i}}$ and $q_{i+1}$ and $q_{i}$ are the two consecutive primes with $\left(q_{i+1}-q_{i}\right)>2$.

By (42.2): $\sum_{i=1}^{\infty}(Q i)^{\sqrt{i}}<\sum_{i=1}^{\infty}\left[\frac{1}{(q i+1-q i)^{i}}{ }^{*} \delta \mathrm{i}\right]+\sum_{i=1}^{\infty}\left(\frac{1}{2^{i}}\right) ;$ because $\max \left\{\delta^{\prime}{ }_{i}\right\}=1$

But $\mathrm{Q}_{\mathrm{i}}=1 /\left[\mathrm{P}_{\mathrm{i}+1}-\mathrm{P}_{\mathrm{i}}\right]^{v_{\mathrm{i}}}$. Consider $\left\{\sum_{i=1}^{\infty} Q i-\left[\sum_{i=1}^{\infty}\left[\delta i * \frac{1}{(q i+1-q i)^{i}}\right]+\sum_{i=1}^{\infty}\left(\frac{1}{2^{i}}\right)\right]\right\}$

But $\sum_{i=1}^{\infty} Q i-\left[\sum_{i=1}^{\infty} \delta i * \frac{1}{(q i+1-q i)^{i}}+\sum_{i=1}^{\infty}\left(\frac{1}{2^{i}}\right)\right]$

$=\sum_{i=1}^{\infty} \frac{1}{(P i+1-P i)^{\sqrt{i}}}-\left[\left[\sum_{i=1}^{\infty} \frac{1}{(q i+1-q i)^{i}} * \delta i\right]+\sum_{i=1}^{\infty}\left(\frac{1}{2^{i}}\right)\right]$

$=\sum_{i=1}^{\infty} \frac{1}{(P i+1-P i)^{\sqrt{ }}}-\sum_{i=1}^{\infty}(Q i)^{\sqrt{ } i}-\sum_{k=1}^{\infty}\left(\frac{1}{2^{k}}\right) \quad($ by 42.2).

Where each $k$ is a natural number where each $k$ generates $\left(P_{k+1}-P_{k}\right) \neq 2$.

Then $\sum_{i=1}^{\infty} \frac{1}{(P i+1-P i)^{\sqrt{ }}}-\sum_{i=1}^{\infty}(Q i)^{\sqrt{ } i}-\sum_{k=1}^{\infty}\left(\frac{1}{2^{k}}\right)$

$>\sum_{i=1}^{\infty} \frac{1}{(P i+1-P i)^{\sqrt{ }}}-\sum_{i=1}^{\infty}(Q i)^{\sqrt{ } i}-\sum_{k=1}^{\infty}\left(\frac{1}{2^{(k-k 0)}}\right)$

Where $k_{0}$ can equals to a natural number (where $k>k_{0}>0$ ) and $k_{0}$ is varying depending on the value of $k$ in each term $\left(P_{k+1}-P_{k}\right)(\neq 2)$. Where, each and every $k_{0}$ generates each $k$ such that $\left(P_{(k-k 0)+1}-P_{(k-k 0)}\right)=2$. 
Then

$\sum_{i=1}^{\infty} \frac{1}{(P i+1-P i)^{\sqrt{ }}}-\sum_{i=1}^{\infty}(Q i)^{\sqrt{ } i}-\sum_{k=1}^{\infty}\left(\frac{1}{2^{k}}\right)>$

$\sum_{i=1}^{\infty} \frac{1}{(P i+1-P i)^{\sqrt{ }}}-\sum_{i=1}^{\infty} \frac{1}{(P i+1-P i)^{i}}-\sum_{k=1}^{\infty}\left(\frac{1}{2^{(k-k 0)}}\right) \quad>\sum_{i=1}^{\infty} \frac{1}{(P i+1-P i)^{\sqrt{ }}}-\sum_{i=1}^{\infty} \frac{2}{(P i+1-P i)^{i}}$ (42.3)

( Because, $\left.\sum_{i=1}^{\infty} \frac{1}{(P i+1-P i)^{i}}\right)>\sum_{k=1}^{\infty}\left(\frac{1}{2^{(k-k 0)}}\right)$, since there are additional terms in $\sum_{i=1}^{\infty} \frac{1}{(P i+1-P i)^{i}}$ compare to $\sum_{k=1}^{\infty}\left(\frac{1}{2^{(k-k 0)}}\right)$, according to the definition of $\left(\mathrm{P}_{\mathrm{i}+1}-\mathrm{P}_{\mathrm{i}}\right)$ and according to the above definition of ( $\left.k-k_{0}\right)$ ).

Then $\sum_{i=1}^{\infty} \frac{1}{(P i+1-P i)^{\sqrt{i}}}-\sum_{i=1}^{\infty} \frac{2}{(P i+1-P i)^{i}}=\sum_{i=1}^{\infty} \frac{(P i+1-P i)^{\sqrt{i}}-2}{(P i+1-P i)^{i}}$. But $\left(\mathrm{P}_{\mathrm{i}+1}-\mathrm{P}_{\mathrm{i}}\right) \geq 2$ and $\mathrm{i} \geq 1$.

Thus $\left(\mathrm{P}_{\mathrm{i}+1}-\mathrm{P}_{\mathrm{i}}\right)^{\sqrt{i}} \geq 2$. Thus $\sum_{i=1}^{\infty} \frac{1}{(P i+1-P i)^{\sqrt{i}}}-\sum_{i=1}^{\infty} \frac{2}{(P i+1-P i)^{i}} \geq 0$.

Thus by (42.3) and by (42.2.1): $\sum_{i=1}^{\infty} Q i-\left[\sum_{i=1}^{\infty} \frac{1}{(q i+1-q i)^{i}} * \delta i+\sum_{i=1}^{\infty}\left(\frac{1}{2^{i}}\right)\right]>0$.

Then $\sum_{i=1}^{\infty} Q i>\left[\sum_{i=1}^{\infty} \frac{1}{(q i+1-q i)^{i}} * \delta i\right]+\left[\sum_{i=1}^{\infty}\left(\frac{1}{2^{i}}\right)\right]>\left[\sum_{i=1}^{\infty}\left(\frac{1}{2^{i}}\right)\right]$

(since $\delta_{i}=1$ whenever $\left(q_{i+1}-q_{i}\right)>2\left\{\right.$ and $\delta_{i}=0$ whenever $\left.\left(P_{i+1}-P_{i}\right)=2\right\}$ and $\frac{1}{(q i+1-q i)^{i}}>$ 0 )

Thus $\sum_{i=1}^{\infty} Q i>\left[\sum_{i=1}^{\infty}\left(\frac{1}{2^{i}}\right)\right]$

(Because by the result (42), there are infinitely many twin prime numbers. Then there are infinitely many $\mathrm{P}_{\mathrm{i}+1}$ and $\mathrm{P}_{\mathrm{i}}$ such that $\left(\mathrm{P}_{\mathrm{i}+1}-\mathrm{P}_{\mathrm{i}}\right)=2$ for infinitely many 'i' natural numbers. Then we can tend $\mathrm{i} \rightarrow$ infinity in the expression $\sum_{i=1}^{\infty}\left(\frac{1}{2^{i}}\right)$ )

Then by (43): $\sum_{i=1}^{\infty} Q i>\sum_{i=1}^{\infty}\left(\frac{1}{2^{i}}\right)$

But we know that $\sum_{i=1}^{\infty}\left(\frac{1}{2^{i}}\right)=1$

By (43), (44) $: \sum_{i=1}^{\infty} Q i>\sum_{i=1}^{\infty}\left(\frac{1}{2^{i}}\right)=1$ 
Thus $\sum_{i=1}^{\infty} Q i>1$

But we know that $Q_{i}$ is the probability of finding the particle $P$ in the quantum state ' $i$ '. But we know that there are infinitely many quantum states in region $A$. That's why we could tend $i$ to infinity. By (45), the total probability of finding the particle $P$, in all of the quantum states 'i'

$=\sum_{i=1}^{\infty} Q i>1$

But we know that although there are infinitely many quantum states in the region $A$, the total probability of the existence of $P$ (arbitrary chosen microscopic particle) in all 'i'th quantum states of region $A$ should be 1.

But the total probability of finding the arbitrarily chosen particle $P$ in the region $A=$ $\sum_{i=1}^{\infty} Q i>1$. Since I chose $\mathrm{P}$ arbitrarily, the total probability of finding $\mathrm{P}$ in the region $\mathrm{A}$ is greater than 1 means there should be some another arbitrary particles those have come from the other region $B$ to the region $A$.

That means: If we can have $Q_{i}=1 /\left[P_{i+1}-P_{i}\right]^{\sqrt{i}}$ for each 'i' eigen state ' $i$ ' ( with two consecutive primes (with $\mathrm{P}_{\mathrm{i}+1}>\mathrm{Pi}_{\mathrm{i}}>\mathrm{N}$; for arbitrary natural number $\mathrm{N}$ greater than 2 and for the considered arbitrary natural number $\mathrm{N}$, the minimum $\mathrm{P}_{\mathrm{i}}$ and $\mathrm{P}_{\mathrm{i}+1}$ obeys $P_{i+1}-P_{i}=2$. i.e. for the chosen $\left.N, \quad\left[P_{2}-P_{1}\right]=2\right)$ ) and ' $i$ ' $\in\{1,2,3, \ldots \ldots, n, \ldots \ldots\}$ ), for arbitrarily chosen microscopic particle $P$ chosen from the region $A$, then there should be some arbitrary particles those have come from the other region $B$ to the region $A$. Those arbitrarily chosen particles $\mathrm{P}$ came from the region $\mathrm{B}$ by penetrating the region $\mathrm{C}$, due to the high thermal density inside the region $B$.

Therefore if there is such a high thermal energy density inside the region $B$ which is increasing gradually during $t \in\left(0, t_{i}\right]$, then those thermal energy radiation will transmit to the region $\mathrm{A}$ little bit (though the region $\mathrm{C}$ ) and then there should be some arbitrary particles those have come from the other region $B$ to the region $A$, if the energies of $P$ allow it to has:

Probability $\left(Q_{i}\right)$ of finding the particle $P$ in the quantum state ' $i$ ' $=\left|C_{i}^{2}\right|$ 
$=\left\{1 /\left[\mathrm{P}_{i+1}-\mathrm{P}_{i}\right]\right\}^{\sqrt{i}} ;$ for $\mathrm{P}_{\mathrm{i}}$ and $\mathrm{P}_{\mathrm{i}+1}$ two arbitrary natural numbers (greater than some arbitrary natural number $\mathrm{N}$ and for the considered arbitrary natural number $\mathrm{N}$, the minimum $P_{i}$ and $P_{i+1}$ obeys $P_{i+1}-P_{i}=2$. i.e. for the chosen $\left.N,\left[P_{2}-P_{1}\right]=2\right)$ ) and for all such $\mathrm{P}_{\mathrm{i}+1}$ and $\mathrm{P}_{\mathrm{i}} ;\left[\mathrm{P}_{\mathrm{i}} / \mathrm{P}_{\mathrm{i}+1}\right]$ is a rational number for any natural number $\mathrm{P}_{\mathrm{i}+1}$ and $\mathrm{P}_{\mathrm{i}}$, greater than the considered arbitrary natural number $\mathrm{N}$.

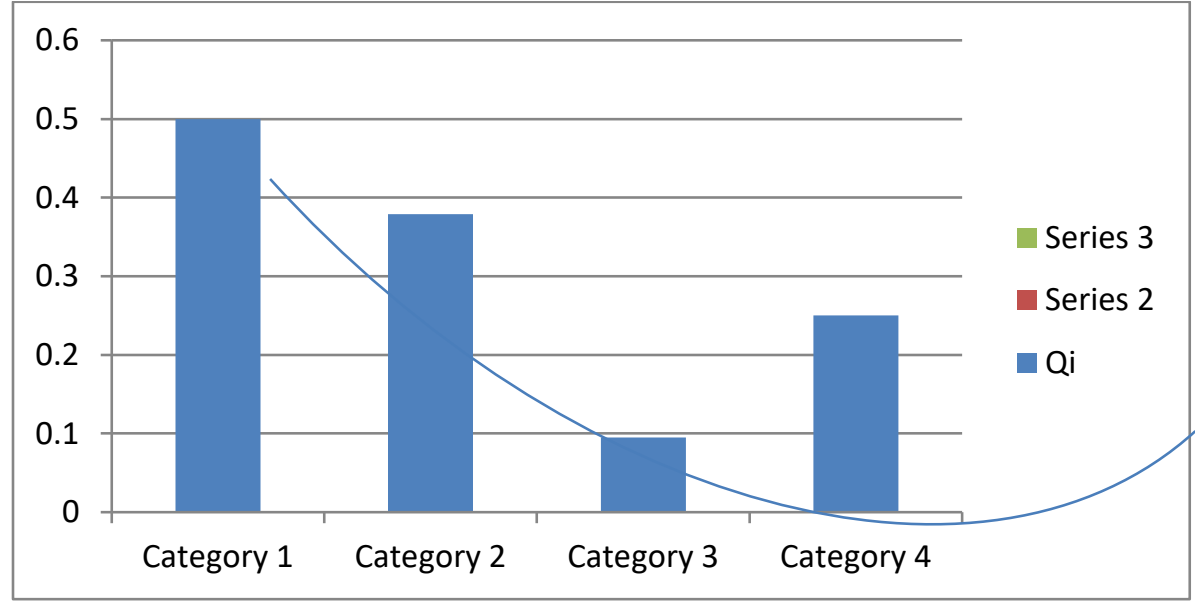

Figure 02 (the variance of $Q_{i}$ vs $\left[P_{i+1}-P_{i}\right]$ ) value of $\left[P_{i+1}-P_{i}\right]$

The $x$ axis of above figure 02 indicates the value of $\left[P_{i+1}-P_{i}\right]$ for different values of ' $i$ '. $Y$ axis indicates the value of $Q_{i}$ for different value of ' $i$ '.

\section{Conclusion}

We assumed initially that there are finitely many twin primes. After proceeding with that, I finally received a contradiction. But in order to get the contradiction, I used that $P_{N}$ as a prime number greater than $\left(P_{R-1}+2\right)$. And also we chose $P_{n}$ odd integer $(>1)$ and also we chose an integer $A_{1}$ such that $P_{3} \mid\left(A_{1}-2\right)$. Also to get the contradiction, I used the fact that $\left(P_{N}-2\right)$ as a non-prime since $\left(P_{N}-2\right)>\left(P_{R-1}+2\right)$. And also I have used that $x_{3}$ as a natural number not equals to 1 (since $\left(\mathrm{P}_{\mathrm{N}}-2\right)$ is not a prime number). And also I have used the fact (to get the contradiction as in (37) ): The difference between any two consecutive prime numbers (which equals to $\left[\left(P_{N}+A_{1}\right)-P_{N}\right]$ ) which is $A_{1}$, is greater 
than $2\left(\right.$ Since $\left[\left(P_{N}+A_{1}\right)\right.$ and $P_{N}$ are greater than $\left.\left(P_{R-1}+2\right)\right)$. i.e. $A_{1} \neq(+/-2)$. Therefore to get the contradiction as in (37), I have used the facts got from our assumption (1). Then the only possibility is our assumption (1) is false. Since our assumption (1) is a proposition, it is possible to state that 'there are infinitely many twin prime numbers'.

Also, if there is a high thermal energy density inside the region B which is increasing gradually during $t \epsilon\left(0, t_{i}\right]$, then those thermal energy radiation will transmit to the region A little bit (though the region $C$ ) and then there should be some arbitrary particles those have come from the other region $B$ to the region $A$, if the energies of $P$ allow it to have $Q_{i}=1 /\left[P_{i+1}-P_{i}\right]^{\sqrt{i}}$ for each ' $i$ ' eigen state ( with two consecutive primes (with $P_{i+1}>P_{i}$ greater than arbitrary natural number $\mathrm{N}$ and for the considered arbitrary natural number $\underline{N}$, the minimum $P_{i}$ and $P_{i+1}$ obeys $P_{i+1}-P_{i}=2$. i.e. for the chosen $\left.N,\left[P_{2}-P_{1}\right]=2\right)$ ) and 'i' $\in\{1,2,3, \ldots \ldots, n, \ldots \ldots$.$\} for arbitrarily chosen microscopic particle$

\section{Appendix}

Prime number: A natural number which divides by 1 and itself only.

Twin Prime Numbers: Two consecutive prime numbers which have the difference exactly 2 .

We denote 'i' th prime gap $g_{i}=P_{i+1}-P_{i}$

Then according to the $2^{\text {nd }}$ reference; Prime number $\mathrm{P}_{\mathrm{N}}=2+\sum_{j=1}^{N-1} g j$

where by $2^{\text {nd }}$ reference: for all $€>0$, there is a natural number ' $n$ ' such that for all $N-1>$ $\mathrm{n} ; \mathrm{g}_{\mathrm{N}-1}<\mathrm{P}_{\mathrm{N}-1} . €$

*** The Time-independent Schrodinger equation can be written as below:

$-\left(\hbar^{2} / 2 m\right) \cdot \nabla^{2} \Psi+V \cdot \Psi=E . \Psi$

Where $\hbar$ is the reduced plank constant, $m$ is the mass of the matter particle ' $P$ ' .

$V$ is the potential energy operator of the system, $E$ is the energy term (eigenvalue). 
And $\Psi$ is the wave function of the matter particle (eigen function).

Where $\nabla^{2}=\frac{1}{r^{2}}\left[\frac{\partial}{\partial r}\left(r^{2} \frac{\partial}{\partial r}\right)+\left(\frac{1}{\sin \theta}\right) \frac{\partial}{\partial \theta}\left(\sin \theta \frac{\partial}{\partial \theta}\right)+\frac{1}{\sin ^{2} \theta} \frac{\partial^{2}}{\partial \varphi^{2}}\right]$

Let $\Phi=\mathrm{C}_{1} . \psi_{1}+\mathrm{C}_{2} . \psi_{2}+$ + Cn. $\psi_{n}$

Where $\mathrm{C}_{i}$ are linearly independent constants $; \mathrm{i} \in\{1,2,3$, $\mathrm{n}$

$\theta$ is the polar angle of considered matter particle $P$ with respect to the origin.

$\varphi$ is the azimuthal angle to the particle $P$ with respect to the origin. $r$ is the distance coordinate to $\mathrm{P}$ with respect to the origin.

$\Phi=\mathrm{C}_{1} \cdot \psi_{1}+\mathrm{C}_{2} . \psi_{2}+\ldots+\mathrm{C}_{n} \cdot \psi_{\mathrm{n}}+\ldots \ldots \ldots \ldots \ldots$ called as a linear combination of $\psi_{\mathrm{i}}$ eigen functions.

If $\psi_{i}$ are orthonormal eigen functions, then probability of finding the particle in the quantum state ' $\mathrm{i}$ ' $=\left|\mathrm{Ci}^{2}\right|$ 


\section{References}

1. Zhang, Yitang (2014). "Bounded gaps between primes". Annals of Mathematics. 179 (3): 1121-1174.

2. https://ipfs.io/ipfs/QmXoypizjW3WknFiJnKLwHCnL72vedxjQkDDP1mXWo6uco/ wiki/Prime_gap.html

3. Terry Tao, Small and Large Gaps between the Primes

4. Maynard, James (2015), "Small gaps between primes", Annals of Mathematics, Second Series, 181 (1): 383-413

5. Tchudakoff, N. G. (1936). "On the difference between two neighboring prime numbers". Math. Sb. 1: 799-814.

6. Ingham, A. E. (1937). "On the difference between consecutive primes" Quarterly Journal of Mathematics. Oxford Series. 8 (1): 255-266.

7. Ralph, R. . Thermal Physics. Cambridge: Cambridge University Press: ISBN 0521-65838-1

8. P. A. M. Dirac, The Quantum Theory of the Emission and Absorption of Radiation, Proc. Royal Soc. Lond. A 114, pp. 243-265, (1927) 